\title{
Two-term Szegó theorem for generalised anti-Wick operators
}

\author{
James P. Oldfield
}

\begin{abstract}
This article concerns the asymptotics of pseudodifferential operators whose Weyl symbol is the convolution of a discontinuous function dilated by a large scaling parameter with a smooth function of constant scale. These operators include as a special case generalised anti-Wick operators, also known as Gabor-Toeplitz operators, with smooth windows and dilated discontinuous symbol. The main result is a two-term Szegó theorem, that is, the asymptotics of the trace of a function of the operator. A special case of this is the asymptotic terms of the eigenvalue counting function. In both cases, previously only the first term in the asymptotic expansion was known explicitly.
\end{abstract}

Mathematics Subject Classification (2010). Primary: 47G30; Secondary: 35P20, 42A38, 47B10, 47B35.

Keywords. Gabor-Toeplitz operators, anti-Wick operators, pseudodifferential operators, Weyl quantisation, Szegố theorem, asymptotic distribution of eigenvalues.

\section{Contents}

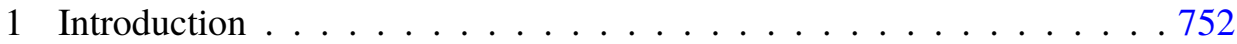

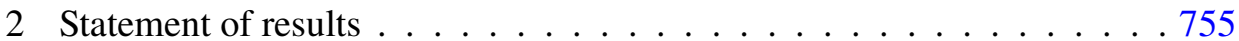

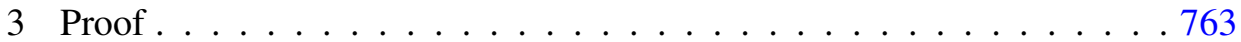

4 Appendix: Tubular neighbourhood properties . . . . . . . . . . . 775

References . . . . . . . . . . . . . . . . . . . . . .780 


\section{Introduction}

We consider pseudodifferential operators whose symbol $p$ is dilated by a large scaling parameter $r$ and "smoothed out" by a convolution factor $W \in \mathcal{S}\left(\mathbb{R}^{2 d}\right)$ whose integral is 1. Explicitly, using the Weyl quantisation (see §2.1), we consider operators acting on $L^{2}\left(\mathbb{R}^{d}\right)$ of the form

$$
T_{r}[p]:=\mathrm{op}\left[W * p_{r}\right], \quad \text { where for } z \in \mathbb{R}^{2 d} \text { we set } p_{r}(z):=p(z / r) .
$$

The main interest in these operators arises from generalised anti-Wick operators. The generalised anti-Wick operator with windows $\varphi_{1}, \varphi_{2} \in L^{2}\left(\mathbb{R}^{d}\right)$ and symbol $p \in L^{\infty}\left(\mathbb{R}^{d}\right)$ is defined to be $\mathcal{F}_{\varphi_{2}}^{*} p \mathcal{F}_{\varphi_{1}}$, where $\mathcal{F}_{\varphi}: L^{2}\left(\mathbb{R}^{d}\right) \rightarrow L^{2}\left(\mathbb{R}^{2 d}\right)$ is the short-time Fourier transform and $p$ acts by multiplication on $L^{2}\left(\mathbb{R}^{2 d}\right)$ (see $\left.\$ 2.4\right)$. These are a special case of operators the form $T_{r}[p]$; that is, for suitable windows $\varphi_{1}, \varphi_{2}$, there is a corresponding $W$ such that

$$
T_{r}[p]=\mathcal{F}_{\varphi_{2}}^{*} p_{r} \mathcal{F}_{\varphi_{1}}, \quad \text { where for } z \in \mathbb{R}^{2 d} \text { we set } p_{r}(z):=p(z / r) .
$$

The result is part of the asymptotic expansion of $\operatorname{tr} f\left(T_{r}\left[a \chi_{\Omega}\right]\right)$ as $r \rightarrow \infty$, where $\Omega \subseteq \mathbb{R}^{2 d}, a$ is a function acting on $\mathbb{R}^{2 d}$ and $f$ is a function such that $f(0)=0$. Since the symbol $a \chi_{\Omega}$ is discontinuous, this is referred to as a Szegótype expansion in analogue with such formulae for Toeplitz matrices. Such a result is already known (discussed below) but only for the first term, which is a standard Weyl-type term of order $r^{2 d}$. The result proved here (see \$2.2) gives an explicit expression for the second asymptotic term, which is a boundary-related term of order $r^{2 d-1}$.

An important special case of this is where $a \equiv 1$ and $f$ is an indicator function, which gives the asymptotics of the eigenvalue counting function of $T_{r}\left[\chi_{\Omega}\right]$. The first term of this expansion shows how many eigenvalues are close to 1 , and the second term shows how many eigenvalues are between 0 and 1 (in what is sometimes called the "plunge region"). This gives some quantitative detail to the idea that $T_{r}\left[\chi_{\Omega}\right]$ acts somewhat like a projection, in that it "projects" the timefrequency representation of functions on to $\Omega$. As with the general result, previously known results about the eigenvalue counting function (discussed below) only give an explicit expression for the first asymptotic term, whereas the result proved here (see §2.3) gives an explicit expression for the second term.

The semiclassical calculus for operators whose Weyl symbol is smooth is already well known [17, Theorem (III-11)]. However, although the Weyl symbol of interest here $W * p_{r}$ is smooth, even when $p$ is discontinuous, it is not of the correct asymptotic form to apply that theory. In the terminology of Robert [17, 
Definition (II-13)], it is not an $h$-admissible operator (with the natural choice of $\left.h=1 / r^{2}\right)$. The problem is that symbol depends upon two different scales in the phase space variable $z$ : when $z$ is far from the boundary of $\Omega,\left(a \chi_{\Omega}\right)_{r}(z)$ varies asymptotically like $a_{r}(z)$, so changes in $z$ proportional to $r$ are important; when $z$ is near to the boundary it varies like $W * \chi_{r \Omega}(z)$, so changes in $z$ on a constant scale are important.

The proof (outlined more precisely in \$3.1) begins in a similar way to that of the usual semiclassical calculus: we prove a Weyl composition result with the usual formula for the approximating symbol, but the remainder is shown to satisfy trace norm and operator norm bounds that are more delicate than usual (Lemma 3.4). The author hopes that these estimates may be of independent interest. In $\$ 3.2$ this result is proved and combined with facts about the geometry of $\Omega$ to show that we may compose $T_{r}\left[a \chi_{\Omega}\right]$ with itself with sufficiently small remainder. In $\S 3.3$ the trace asymptotics of the resulting operator are established using further geometrical facts. The relevant geometrical theory of tubular neighbourhoods is collected in $\S 4$.

Related Szegó-type theorems. The original Szegó theorems are results about the asymptotic expansion of $\log \operatorname{det} T_{n}$ (that is, $\operatorname{tr} \log T_{n}$ ) as $n \rightarrow \infty$, where $T_{n}$ is an $n \times n$ Toeplitz matrix [22, 23]; see also [12, Chapter 5]. (The parameter $r$ used here is analogous to $n^{2}$ in such problems.) Similar theorems have been proved for Wiener-Hopf operators, which are a continuous analogue of Toeplitz operators: whereas Toeplitz operators involve discrete convolution with a sequence and truncation to a finite length, Wiener-Hopf operators involve the standard convolution with a function and truncation to a bounded domain. Szegő theorems for both types of operator have been the subject of extensive study; see, for example, [3]. The intention here is just to highlight a few of the most directly relevant results.

A generalization of Wiener-Hopf operators is pseudodifferential operators with discontinuous symbol (without the convolution factor $W$ as in the operators considered here, and usually with the left quantisation rather than the Weyl quantisation). If $\widetilde{T}_{r}$ is a pseudodifferential operator with symbol of the form $a(\boldsymbol{x}, \boldsymbol{\xi}) \chi_{\Omega}(\boldsymbol{x})$ where $a$ is smooth, i.e. the discontinuity is in the configuration variable but not the frequency variable, the complete asymptotic expansion of $\operatorname{tr} f\left(\widetilde{T}_{r}\right)$ is known for quite general functions [25]. The terms in this expansion are of the order $r^{2(d-k)}$, where $k$ takes non-negative integer values. The coefficients depend on the geometry of $\Omega$, and it is possible to obtain geometrical insights into these coefficients $[18,19]$ by using geometrical ideas broadly similar to the ones used in this paper, particularly Lemma 4.8 . 
When there is also a discontinuity in the frequency variable, i.e. the symbol is of the form $a \chi_{\Omega_{1} \times \Omega_{2}}$ where $\Omega_{1}, \Omega \subseteq \mathbb{R}^{d}$, two terms of the asymptotic expansion of $\operatorname{tr} f\left(\widetilde{T}_{r}\right)$ are known (proved by Widom [24] in one dimension and Sobolev $[21,20]$ in higher dimensions). The first term is equal to the one in the result proved here (in particular it is of order $r^{2 d}$ ). However, the second term is of order $r^{2 d-2} \log r$ and depends on the value of $a$ on $\partial \Omega_{1} \times \partial \Omega_{2}$, in contrast to the result proved here where the second term is of order $r^{2 d-1}$ and depends on the value of $a$ on $\partial \Omega$.

For generalised anti-Wick operators, which are a subclass of the operators $T_{r}[p]$ considered here, a one-term Szegó theorem was found by Feichtinger and Nowak [8]. (They called these operators Gabor-Toeplitz operators.) Compared to the requirements here, their regularity requirements are very mild: the symbol merely has to be in $L^{1} \cap L^{\infty}$, rather than possessing a discontinuity of the specific form $\chi_{\Omega}$, and the window function merely has to be in $L^{2}\left(\mathbb{R}^{d}\right)$ rather than $\mathcal{S}\left(\mathbb{R}^{d}\right)$. However the symbol must also be positive and the two windows must be equal, which implies that the operator is positive. That result is for the first term in the asymptotic expansion, with $o\left(r^{2 d}\right)$ remainder.

Related eigenvalue counting function results. The asymptotics of the eigenvalue counting function is a consequence of the Szegó theorem for $T_{r}\left[\chi_{\Omega}\right]$, but has also been studied in its own right.

Anti-Wick operators (which are generalised anti-Wick operators with Gaussian windows) were first studied systematically by Berezin [1]. This included a result (Theorem 12 of that paper) giving one asymptotic term of the eigenvalue counting function in roughly the inverse situation to the one of interest here: he considered eigenvalues below a fixed value, for symbols that are bounded below by a positive value.

Anti-Wick operators were introduced into the time-frequency community by a paper of Daubechies [5], which she called time-frequency localization operators when the symbol is an indicator function. This included two asymptotic terms of the eigenvalue counting function (Remark 2 and Remark 3 in §IV.B of that paper) for a specific operator: the anti-Wick operator whose symbol is the indicator function of the unit disc. She proved this by explicitly finding the eigenvalues and eigenfunctions of this operator, using the fact that these are known for Weyl pseudodifferential operators with spherically symmetric symbols.

For generalised anti-Wick operators whose symbol is a general indicator function, only the first asymptotic term of the eigenvalue counting function was previously known. This was shown for one dimensional operators by Ramanathan and 
Topiwala [16, Theorem 2 and Corollary 1], and in higher dimensions by Feichtinger and Nowak [8, Corollary 2.3 and Comment (iii) in §2] using their Szegố result. De Mari, Feichtinger and Nowak [6, Example (a) on p. 731] showed that the asymptotic order of the second term is $r^{2 d-1}$ (including a lower bound for it), but did not find an explicit expression.

Notation. Here are a few notational conventions used throughout. We denote the space of Schwartz functions on $\mathbb{R}^{m}$ by $\mathcal{S}\left(\mathbb{R}^{m}\right)$. The function $\chi_{\Lambda}$ is the indicator function of a set $\Lambda$. We denote the $k$-dimensional Hausdorff measure by $\mu_{k}$; in particular $\mu_{m-1}(\mathrm{~d} \boldsymbol{u})$ is the surface element in $\mathbb{R}^{m}$, and when $k$ equals the ambient dimension $\mu_{k}$ is simply the Lebesgue measure. The set of natural numbers including zero is denoted by $\mathbb{N}_{0}$, so that the set of $m$-dimensional multi-indices is $\mathbb{N}_{0}^{m}$. The boundary of a set $\Omega$ is denoted by $\partial \Omega$ and its complement by $\Omega^{\mathrm{c}}$. The tubular radius $\tau(\partial \Omega)$ and tubular neighbourhood $\operatorname{tub}(\partial \Omega, t)$ of $\partial \Omega$ are defined in $\S 4.1$.

Acknowledgements. It is the author's pleasure to thank A.V. Sobolev for suggesting the problem and his tireless support, especially his observation that a rougher version of Lemma 3.4 (similar to [21, Lemma 3.12 and Corollary 3.13]) could give the asymptotics when $\partial \Omega$ is straight.

The author would also like to thank the organisers of the workshop on phase space methods for pseudodifferential operators at the Erwin Schrödinger Institute in October 2012, where he had many productive discussions. This included a conversation with K. Nowak, who the author would like to thank for informing him of a two-term Szegó theorem that K. Nowak and H. G. Feichtinger have made progress on under somewhat different conditions to those considered here.

This work was supported by the Engineering and Physical Sciences Research Council [grant number EP/P505771/1].

\section{Statement of results}

2.1. Weyl quantisation preliminaries. We will use the Weyl quantisation: for a suitable symbol $q$, we define the operator op $[q]$ for each $u \in \mathcal{S}\left(\mathbb{R}^{2 d}\right)$ by

$$
(\operatorname{op}[q] u)(\boldsymbol{x}):=\frac{1}{(2 \pi)^{d}} \int_{\mathbb{R}^{d}} \int_{\mathbb{R}^{d}} \mathrm{e}^{\mathrm{i}(\boldsymbol{x}-\boldsymbol{y}) \cdot \boldsymbol{\xi}} q\left(\frac{1}{2}(\boldsymbol{x}+\boldsymbol{y}), \boldsymbol{\xi}\right) u(\boldsymbol{y}) \mathrm{d} \boldsymbol{y} \mathrm{d} \boldsymbol{\xi},
$$


and extend this to $L^{2}\left(\mathbb{R}^{d}\right)$ by density. This satisfies the operator norm and trace norm estimates ([4, Corollary 2.5(i)] and [7, Theorem 9.4] respectively)

$$
\|\operatorname{op}[q]\| \leqslant C_{d} \sum_{|\alpha| \leqslant d+2}\left\|\partial^{\alpha} q\right\|_{L^{\infty}\left(\mathbb{R}^{2 d}\right)}, \quad\|\operatorname{op}[q]\|_{1} \leqslant C_{d}^{\prime} \sum_{|\alpha| \leqslant 2 d+1}\left\|\partial^{\alpha} q\right\|_{L^{1}\left(\mathbb{R}^{2 d}\right)},
$$

where $C_{d}$ and $C_{d}^{\prime}$ are constants depending only on the dimension and the sums are taken over multi-indices $\alpha \in \mathbb{N}_{0}^{2 d}$. (This operator norm estimate is slightly weaker than the one in the cited work, but is sufficient for our purposes.) When the trace norm estimate is finite, the trace exists and equals

$$
\operatorname{trop}[q]=\frac{1}{(2 \pi)^{d}} \int_{\mathbb{R}^{2 d}} q(z) \mathrm{d} z .
$$

The adjoint of the operator is given by [9, Proposition (2.6)]

$$
(\mathrm{op}[q])^{*}=\mathrm{op}[\bar{q}] ;
$$

in particular, if $q$ is real-valued then $\mathrm{op}[q]$ is self-adjoint.

As stated in the introduction, the operators of interest here depend on a discontinuous symbol $p$, dilated by a factor $r$ and convolved with a Schwartz function $W \in \mathcal{S}\left(\mathbb{R}^{2 d}\right)$, so that

$$
T_{r}[p]:=\mathrm{op}\left[W * p_{r}\right], \quad \text { where for } z \in \mathbb{R}^{2 d} \text { we set } p_{r}(z):=p(z / r) .
$$

Applying the Weyl operator norm and trace norm estimates to $T_{r}[p]$ we obtain

$$
\begin{gathered}
\left\|T_{r}[p]\right\| \leqslant C_{d} \sum_{|\alpha| \leqslant d+2}\left\|\partial^{\alpha} W\right\|_{L^{1}\left(\mathbb{R}^{2 d}\right)}\|p\|_{L^{\infty}\left(\mathbb{R}^{2 d}\right)}, \\
\left\|T_{r}[p]\right\|_{1} \leqslant C_{d}^{\prime} r^{2 d} \sum_{|\alpha| \leqslant 2 d+1}\left\|\partial^{\alpha} W\right\|_{L^{1}\left(\mathbb{R}^{2 d}\right)}\|p\|_{L^{1}\left(\mathbb{R}^{2 d}\right)} .
\end{gathered}
$$

Since we will be interested in the effects of varying the scale of the discontinuous part of the symbol, rather than varying $W$, we will often use the notation

$$
x \lesssim y \quad \Longleftrightarrow \quad \text { there exists } C_{W}>0 \text { such that } x \leqslant C_{W} y,
$$

where $C_{W}$ is some constant depending only on $W$ and the dimension $d$ (not on $p$ or $r$ ). Using this notation, the above inequalities are

$$
\left\|T_{r}[p]\right\| \lesssim\|p\|_{L^{\infty}\left(\mathbb{R}^{2 d}\right)}, \quad\left\|T_{r}[p]\right\|_{1} \lesssim r^{2 d}\|p\|_{L^{1}\left(\mathbb{R}^{2 d}\right)} .
$$

The trace formula, combined with the fact that the integral of $W$ is 1 , gives

$$
\operatorname{tr} T_{r}[p]=\frac{r^{2 d}}{(2 \pi)^{d}} \int_{\mathbb{R}^{2 d}} p(z) \mathrm{d} z .
$$


2.2. Szegó theorem. In this subsection we state Theorem 2.4, the Szegó theorem for operators of the form $T_{r}\left[a \chi_{\Omega}\right]$. It has the following regularity conditions on the symbol.

Condition 2.1. Let all of the following be satisfied.

- Let $W \in \mathcal{S}\left(\mathbb{R}^{2 d}\right)$ satisfy $\int_{\mathbb{R}^{2 d}} W(z) \mathrm{d} z=1$.

- Let $\Omega \subseteq \mathbb{R}^{2 d}$ have a boundary that is $C^{2}$ and has a tubular neighbourhood (see $\$ 4)$.

- Let $a$ be a twice continuously differentiable function on $\mathbb{R}^{2 d}$ satisfying $\partial^{\alpha} a \in L^{1}\left(\mathbb{R}^{2 d}\right) \cap L^{\infty}\left(\mathbb{R}^{2 d}\right)$ for all $\alpha \in \mathbb{N}_{0}^{2 d}$ such that $|\alpha| \leqslant 2$.

Remark 2.2. Whenever Condition 2.1 is satisfied we can conclude that that $a$ satisfies the boundary integrability properties $\partial^{\alpha} a \in L^{1}(\partial \Omega)$ for $|\alpha| \leqslant 1$. This can be seen by applying Lemma 4.11 with $g \equiv 1$.

We also need a condition on the regularity of $f$. This depends on whether we define $f\left(T_{r}\left[a \chi_{\Omega}\right]\right)$ using the holomorphic functional calculus or the Borel functional calculus. In the latter case we impose additional restrictions on $W$ and $a$ to ensure that the operator $T_{r}\left[a \chi_{\Omega}\right]$ is self-adjoint (by ensuring that its Weyl symbol is real).

Condition 2.3. For functions $a$ and $W$, let $f$ be a function satisfying $f(0)=0$ and one of the following.

(1) Let $f$ be a holomorphic function on $\mathbb{C}$.

(2) Let $a$ be real-valued, let $W$ be real-valued and let $f$ be an infinitely differentiable function on $\mathbb{R}$.

The boundary term depends on a type of directional antiderivative of $W$. Specifically, for any $W \in \mathcal{S}\left(\mathbb{R}^{2 d}\right)$ with $\int_{\mathbb{R}^{2 d}} W(z) \mathrm{d} z=1$, we define

$$
Q_{\omega}(\lambda):=\int_{\left\{z \in \mathbb{R}^{2 d}: z \cdot \omega \leqslant \lambda\right\}} W(z) \mathrm{d} z \quad\left(\omega \in \mathbb{S}^{2 d-1}\right) .
$$

This satisfies $\lim _{\lambda \rightarrow \infty} Q_{\omega}(\lambda)=\int_{\mathbb{R}^{2 d}} W(z) \mathrm{d} z=1$, and so

$$
1-Q_{\omega}(\lambda)=\int_{\left\{z \in \mathbb{R}^{2 d}: z \cdot \omega \geqslant \lambda\right\}} W(z) \mathrm{d} z \quad\left(\omega \in \mathbb{S}^{2 d-1}\right) .
$$


Theorem 2.4. Let $W, a, \Omega, f$ satisfy Condition 2.1 and Condition 2.3. Then

$\operatorname{tr} f\left(T_{r}\left[a \chi_{\Omega}\right]\right)=r^{2 d} A_{0}(a, \Omega, f)+r^{2 d-1} A_{1}(a, \Omega, f)+O\left(r^{2 d-2}\right)$

as $r \rightarrow \infty$, where

$$
\begin{aligned}
& A_{0}(a, \Omega, f)=\frac{1}{(2 \pi)^{d}} \int_{\Omega} f(a(z)) \mathrm{d} z, \\
& A_{1}(a, \Omega, f)=\frac{1}{(2 \pi)^{d}} \int_{\partial \Omega} \int_{\mathbb{R}}\left(f\left(Q_{\boldsymbol{n}(\boldsymbol{u})}(\lambda) a(\boldsymbol{u})\right)\right. \\
& \left.-Q_{\boldsymbol{n}(\boldsymbol{u})}(\lambda) f(a(\boldsymbol{u}))\right) \mathrm{d} \lambda \mu_{2 d-1}(\mathrm{~d} \boldsymbol{u}) .
\end{aligned}
$$

The proof is given in $\S 3$, including an overview in $\S 3.1$. In the case of generalised anti-Wick operators, the conditions and conclusions can be explicitly expressed in terms of the windows instead of $W$; see $\$ 2.4$.

We now observe why the quantities in Theorem 2.4 are well defined. The operator $f\left(T_{r}\left[a \chi_{\Omega}\right]\right)$ is trace class since, using the fact that $f(0)=0$, we have

$$
\left\|f\left(T_{r}\left[a \chi_{\Omega}\right]\right)\right\|_{1} \leqslant\left\|T_{r}\left[a \chi_{\Omega}\right]\right\|_{1} \sup _{|t| \leqslant\left\|T_{r}\left[a \chi_{\Omega}\right]\right\|}\left|f^{\prime}(t)\right|,
$$

and the bounds in $\S 2.1$ show that this is finite. Let

$$
Q_{\max }:=\sup _{\omega \in \mathbb{S}^{2} d-1} \sup _{\lambda \in \mathbb{R}}\left|Q_{\omega}(\lambda)\right|,
$$

which in particular satisfies $Q_{\max } \leqslant \int_{\mathbb{R}^{2 d}}|W(z)| \mathrm{d} z$. The two asymptotic terms are absolutely integrable with bounds

$$
\begin{aligned}
& \left|A_{0}(a, \Omega, f)\right| \leqslant \frac{1}{(2 \pi)^{d}}\|a\|_{L^{1}(\Omega)} \sup _{|t| \leqslant\|a\|_{L^{\infty}(\Omega)}}\left|f^{\prime}(t)\right|, \\
& \left|A_{1}(a, \Omega, f)\right| \leqslant \frac{2}{(2 \pi)^{d}}\|a\|_{L^{1}(\partial \Omega)} \int_{\mathbb{R}^{2 d}}\left|z^{\prime} W\left(z^{\prime}\right)\right| \mathrm{d} z^{\prime} \sup _{|t| \leqslant Q_{\max }\|a\|_{L^{\infty}(\partial \Omega)}}\left|f^{\prime}(t)\right| .
\end{aligned}
$$

The bound on $A_{0}$ is immediate, and the bound on $A_{1}$ uses the easily checked fact that for any $\omega \in \mathbb{S}^{2 d-1}$ we have

$$
\int_{\mathbb{R}}\left|Q_{\omega}(\lambda)-\chi_{[0, \infty)}(\lambda)\right| \mathrm{d} \lambda \leqslant \int_{\mathbb{R}^{2 d}}\left|\omega \cdot z^{\prime} W\left(z^{\prime}\right)\right| \mathrm{d} z^{\prime} .
$$

2.3. Eigenvalue counting function. In this subsection we give a precise statement of the special case discussed in the introduction: two terms of the asymptotic expansion of the eigenvalue counting function for operators of the form $T_{r}\left[\chi_{\Omega}\right]$. We use the notation $N\left(T_{r}\left[\chi_{\Omega}\right],[\delta, \infty)\right.$ ) to mean the number of eigenvalues of $T_{r}\left[\chi_{\Omega}\right]$ in the interval $[\delta, \infty)$. The proof is a standard approximation argument applied to Theorem 2.4, and is detailed at the end of this subsection. 
Corollary 2.5. Let $\Omega \subseteq \mathbb{R}^{2 d}$ be a compact set with $C^{2}$ boundary and $\delta \in(0,1)$. Let $W \in \mathcal{S}\left(\mathbb{R}^{2 d}\right)$ be real valued and satisfy $\int_{\mathbb{R}^{2 d}} W(z) \mathrm{d} z=1$ and

$$
\mu_{1}\left(\left\{\lambda \in \mathbb{R}: Q_{\omega}(\lambda)=\delta\right\}\right)=0 \quad \text { for all } \omega \in \mathbb{S}^{2 d-1} .
$$

Then

$$
N\left(T_{r}\left[\chi_{\Omega}\right],[\delta, \infty)\right)=r^{2 d} A_{0}\left(1, \Omega, \chi_{[\delta, \infty)}\right)+r^{2 d-1} A_{1}\left(1, \Omega, \chi_{[\delta, \infty)}\right)+o\left(r^{2 d-1}\right)
$$

as $r \rightarrow \infty$. Specifically, these terms satisfy

$$
\begin{aligned}
& A_{0}\left(1, \Omega, \chi_{[\delta, \infty)}\right)=\frac{1}{(2 \pi)^{d}} \mu_{2 d}(\Omega), \\
& A_{1}\left(1, \Omega, \chi_{[\delta, \infty)}\right)=\frac{1}{(2 \pi)^{d}} \int_{\partial \Omega} g_{\boldsymbol{n}(\boldsymbol{u})}(\delta) \mu_{2 d-1}(\mathrm{~d} \boldsymbol{u}),
\end{aligned}
$$

where for each $\delta \in(0,1), \omega \in \mathbb{S}^{2 d-1}$ we set

$$
g_{\omega}(\delta):=\mu_{1}\left(\left\{\lambda \in(-\infty, 0]: Q_{\omega}(\lambda)>\delta\right\}\right)-\mu_{1}\left(\left\{\lambda \in[0, \infty): Q_{\omega}(\lambda)<\delta\right\}\right) .
$$

Remark 2.6. The statement of Corollary 2.5 is somewhat simpler when $Q_{\omega}$ is a non-decreasing function for all $\omega \in \mathbb{S}^{2 d-1}$. A sufficient condition for this is that $W$ is non-negative; for another sufficient condition see Remark 2.8. In this case:

- The condition relating $Q_{\omega}$ and $\delta$ holds if and only if for each $\omega \in \mathbb{S}^{2 d-1}$ there exists a unique $\lambda \in \mathbb{R}$ such that $Q_{\omega}(\lambda)=\delta$; we denote such a $\lambda$ by $Q_{\omega}^{-1}(\delta)$, even if $Q_{\omega}$ is not invertible on its whole domain.

- We then have $g_{\omega}(\delta)=-Q_{\omega}^{-1}(\delta)$, so the boundary term simplifies to

$$
A_{1}\left(1, \Omega, \chi_{[\delta, \infty)}\right)=-\frac{1}{(2 \pi)^{d}} \int_{\partial \Omega} Q_{\boldsymbol{n}(\boldsymbol{u})}^{-1}(\delta) \mu_{2 d-1}(\mathrm{~d} \boldsymbol{u}) .
$$

Proof of Corollary 2.5. We have $N\left(T_{r}\left[\chi_{\Omega}\right],[\delta, \infty)\right)=\operatorname{tr} \chi_{[\delta, \infty)}\left(T_{r}\left[\chi_{\Omega}\right]\right)$; however, we cannot immediately apply Theorem 2.4 with $f:=\chi_{[\delta, \infty)}$ because this function is not sufficiently smooth to satisfy Condition 2.3.

Let $\varepsilon>0$ such that $\varepsilon<\delta$. Let $f_{-\varepsilon}$ and $f_{+\varepsilon}$ be smooth increasing functions satisfying $f_{ \pm \varepsilon}(t)=\chi_{[\delta, \infty)}(t)$ except when $t \in(\delta, \delta+\varepsilon)$ and $t \in(\delta-\varepsilon, \delta)$ respectively. Thus $0 \leqslant f_{-\varepsilon} \leqslant \chi_{[\delta, \infty)} \leqslant f_{+\varepsilon} \leqslant 1$ and

$$
\operatorname{tr} f_{-\varepsilon}\left(T_{r}\left[\chi_{\Omega}\right]\right) \leqslant \operatorname{tr} \chi_{[\delta, \infty)}\left(T_{r}\left[\chi_{\Omega}\right]\right) \leqslant \operatorname{tr} f_{+\varepsilon}\left(T_{r}\left[\chi_{\Omega}\right]\right) .
$$


Applying Theorem 2.4 to $f_{ \pm \varepsilon}\left(T_{r}\left[\chi_{\Omega}\right]\right)$ (with $a \equiv 1$ ), we have

$$
\begin{aligned}
& \lim _{r \rightarrow \infty} \frac{\operatorname{tr}\left(\left(f_{+\varepsilon}-f_{-\varepsilon}\right)\left(T_{r}\left[\chi_{\Omega}\right]\right)\right)}{r^{2 d-1}} \\
& \quad=\frac{1}{(2 \pi)^{d}} \int_{\partial \Omega} \int_{\mathbb{R}}\left(f_{+\varepsilon}\left(Q_{\boldsymbol{n}(\boldsymbol{u})}(\lambda)\right)-f_{-\varepsilon}\left(Q_{\boldsymbol{n}(\boldsymbol{u})}(\lambda)\right)\right) \mathrm{d} \lambda \mu_{2 d-1}(\mathrm{~d} \boldsymbol{u}) \\
& \quad \leqslant \frac{1}{(2 \pi)^{d}} \mu_{2 d-1}(\partial \Omega) \sup _{\boldsymbol{u} \in \partial \Omega} \mu_{1}\left(\left\{\lambda \in \mathbb{R}: \delta-\varepsilon \leqslant Q_{\boldsymbol{n}(\boldsymbol{u})}(\lambda) \leqslant \delta+\varepsilon\right\}\right) .
\end{aligned}
$$

The limit of this bound is 0 as $\varepsilon \rightarrow 0$, so the result follows.

It remains to show that $A_{1}$ satisfies the given form. First set

$$
\tilde{A}_{1}:=\frac{1}{(2 \pi)^{d}} \int_{\partial \Omega} \int_{\mathbb{R}}\left(\chi_{[\delta, \infty)}\left(Q_{\boldsymbol{n}(\boldsymbol{u})}(\lambda)\right)-\chi_{[0, \infty)}(\lambda)\right) \mathrm{d} \lambda \mu_{2 d-1}(\mathrm{~d} \boldsymbol{u}) .
$$

A straightforward calculation shows that

$$
A_{1}-\tilde{A}_{1}=\frac{1}{(2 \pi)^{d}} \int_{\partial \Omega} \boldsymbol{n}(\boldsymbol{u}) \mu_{2 d-1}(\mathrm{~d} \boldsymbol{u}) \cdot \int_{\mathbb{R}^{2 d}} \boldsymbol{z}^{\prime} W\left(\boldsymbol{z}^{\prime}\right) \mathrm{d} \boldsymbol{z}^{\prime},
$$

which by the divergence theorem is zero. It is easily seen that $\widetilde{A}_{1}$ satisfies the stated form.

2.4. Generalised anti-Wick operators. Define the short-time Fourier transform with window $\varphi \in L^{2}\left(\mathbb{R}^{d}\right)$ by

$$
\mathcal{F}_{\varphi}: L^{2}\left(\mathbb{R}^{d}\right) \rightarrow L^{2}\left(\mathbb{R}^{2 d}\right), \quad \mathcal{F}_{\varphi} u(\boldsymbol{x}, \boldsymbol{\xi}):=\frac{1}{(2 \pi)^{d / 2}} \int_{\mathbb{R}^{d}} \mathrm{e}^{-\mathrm{i} \boldsymbol{y} \cdot \boldsymbol{\xi}} u(\boldsymbol{y}) \overline{\varphi(\boldsymbol{y}-\boldsymbol{x})} \mathrm{d} \boldsymbol{y} .
$$

When the window is Gaussian, $\mathcal{F}_{\varphi}$ is also known as the Fourier-Bros-Iagolnitzer transform. (See, for example, [13, Chapter 3] or [14, §3.1] for more information.) The generalised anti-Wick operator with symbol $p$ and windows $\varphi_{1}, \varphi_{2}$ is defined to be $\mathcal{F}_{\varphi_{2}}^{*} p \mathcal{F}_{\varphi_{1}}$. These operators are known under several names, including Gabor-Toeplitz operators, short-time Fourier transform multipliers and time-frequency localization operators. The case where $\varphi_{1}=\varphi_{2}$ is most often of interest.

Generalised anti-Wick operators are bounded on $L^{2}\left(\mathbb{R}^{d}\right)$ when $p \in L^{\infty}\left(\mathbb{R}^{2 d}\right)$ and $\varphi_{1}, \varphi_{2} \in L^{2}\left(\mathbb{R}^{2 d}\right)$. Furthermore, if $p$ is constant then the operator is a multiple of the identity. Specifically,

$$
\begin{gathered}
\left\|\mathcal{F}_{\varphi_{2}}^{*} p \mathcal{F}_{\varphi_{1}}\right\| \leqslant\left\|\varphi_{1}\right\|_{L^{2}\left(\mathbb{R}^{d}\right)}\left\|\varphi_{2}\right\|_{L^{2}\left(\mathbb{R}^{d}\right)}\|p\|_{L^{\infty}\left(\mathbb{R}^{2 d}\right)}, \\
\mathcal{F}_{\varphi_{2}}^{*} \mathcal{F}_{\varphi_{1}}=\left\langle\varphi_{2}, \varphi_{1}\right\rangle_{L^{2}\left(\mathbb{R}^{d}\right)} \operatorname{Id}_{L^{2}\left(\mathbb{R}^{d}\right)} .
\end{gathered}
$$


These relationships can easily be proved from the Fourier inversion theorem, or see for example [13, Corollary 3.2.2 and Corollary 3.2.3].

Theorem 2.4 and Corollary 2.5 apply to generalised anti-Wick operators; that is, there exists a suitable $W$ (depending on the windows) such that

$$
T_{r}[p]=\mathcal{F}_{\varphi_{2}}^{*} p_{r} \mathcal{F}_{\varphi_{1}}, \quad \text { where for } z \in \mathbb{R}^{2 d} \text { we set } p_{r}(z):=p(z / r) .
$$

The following two remarks explain how all references to $W$ in these results may be replaced by references directly to the windows. Afterwards we will describe this $W$ and explain why the remarks are true.

Remark 2.7. The conditions on $W$ can be replaced by requirements on the window functions:

- For all the conditions on $W$ in Theorem 2.4 and Corollary 2.5 to hold (including that $W$ is real-valued), it suffices that $\varphi_{1}=\varphi_{2}$ (which we write simply as $\varphi), \varphi \in \mathcal{S}\left(\mathbb{R}^{2 d}\right)$, and $\|\varphi\|_{L^{2}\left(\mathbb{R}^{d}\right)}=1$.

- For the conditions on $W$ in Theorem 2.4 to hold except that $W$ be real-valued (so we require Condition 2.3(1), the holomorphic $f$ case), it suffices that $\varphi_{1}, \varphi_{2} \in \mathcal{S}\left(\mathbb{R}^{2 d}\right)$ and $\left\langle\varphi_{2}, \varphi_{1}\right\rangle_{L^{2}\left(\mathbb{R}^{d}\right)}=1$.

Remark 2.8. It is possible to express $Q_{\omega}$ directly in terms of the windows. First consider the one-dimensional case. We will use the fractional Fourier transform $\mathcal{F}^{t}$, defined for $t \in \mathbb{R}$ using the functional calculus for unitary operators; thus $\mathcal{F}^{0}=\mathcal{F}^{4}=\operatorname{Id}_{L^{2}(\mathbb{R})}$ and $\mathcal{F}^{1}$ is the usual Fourier transform. We can instead index by direction $\omega \in \mathbb{S}^{1}$, so that $\mathcal{F}^{(1,0)}=\operatorname{Id}_{L^{2}(\mathbb{R})}$ and $\mathcal{F}^{(0,1)}=\mathcal{F}$. The expression for $Q_{\omega}$ is

$$
Q_{\omega}(\lambda)=\int_{-\infty}^{\lambda} \mathcal{F}^{\omega} \varphi_{2}(\eta) \overline{\mathcal{F}^{\omega} \varphi_{1}(\eta)} \mathrm{d} \eta .
$$

In the higher-dimensional case, for each $\omega \in \mathbb{S}^{2 d-1}$ there exists a unitary operator $T_{\boldsymbol{\omega}}$ and $\tilde{\boldsymbol{\omega}} \in \mathbb{S}^{d-1}$ such that

$$
Q_{\omega}(\lambda)=\int_{-\infty}^{\lambda} \int_{\left\{\boldsymbol{x} \in \mathbb{R}^{d}: \boldsymbol{x} \cdot \tilde{\boldsymbol{\omega}}=\eta\right\}} T_{\boldsymbol{\omega}} \varphi_{2}(\boldsymbol{x}) \overline{T_{\boldsymbol{\omega}} \varphi_{1}(\boldsymbol{x})} \mu_{d-1}(\mathrm{~d} \boldsymbol{x}) \mathrm{d} \eta .
$$

In particular, for any dimension, if $\varphi_{1}=\varphi_{2}$ then $Q_{\omega}$ is a non-decreasing function and Remark 2.6 applies.

The key fact that allows us to apply Theorem 2.4 and Corollary 2.5 to generalised anti-Wick operators is their connection to the Weyl transform, given by

$$
\mathcal{F}_{\varphi_{2}}^{*} p \mathcal{F}_{\varphi_{1}}=\operatorname{op}\left[\mathcal{W}_{\varphi_{2}, \varphi_{1}} * p\right],
$$


where

$$
\mathcal{W}_{\varphi_{2}, \varphi_{1}}(\boldsymbol{x}, \boldsymbol{\xi})=\frac{1}{(2 \pi)^{d}} \int_{\mathbb{R}^{d}} \mathrm{e}^{-\mathrm{i} \boldsymbol{t} \cdot \boldsymbol{\xi}} \varphi_{2}\left(\boldsymbol{x}+\frac{1}{2} \boldsymbol{t} \overline{\varphi_{1}\left(\boldsymbol{x}-\frac{1}{2} \boldsymbol{t}\right)} \mathrm{d} \boldsymbol{t} .\right.
$$

The function $\mathcal{W}_{\varphi_{2}, \varphi_{1}}$ is called the Wigner transform of $\varphi_{2}, \varphi_{1}$. This relationship can be found for example in [9, Proposition (3.5)] when $\varphi_{1}=\varphi_{2}$ or [2, Lemma 2.4].

Proof of Remark 2.7. We use the following properties of $\mathcal{W}_{\varphi_{2}, \varphi_{1}}$.

(1) If $\varphi_{1}, \varphi_{2} \in \mathcal{S}\left(\mathbb{R}^{2 d}\right)$ then $\mathcal{W}_{\varphi_{2}, \varphi_{1}} \in \mathcal{S}\left(\mathbb{R}^{2 d}\right)$.

(2) For all $\boldsymbol{x} \in \mathbb{R}^{d}$ we have $\int_{\mathbb{R}^{d}} \mathcal{W}_{\varphi_{2}, \varphi_{1}}(\boldsymbol{x}, \boldsymbol{\xi}) \mathrm{d} \boldsymbol{\xi}=\varphi_{2}(\boldsymbol{x}) \overline{\varphi_{1}(\boldsymbol{x})}$.

(3) We have $\mathcal{W}_{\varphi_{2}, \varphi_{1}}(z)=\overline{\mathcal{W}_{\varphi_{1}, \varphi_{2}}(z)}$.

These properties follow easily from the definition of $\mathcal{W}_{\varphi_{2}, \varphi_{1}}$ (see for example [9, $\S 1.8])$. Remark 2.7 is an immediate consequence.

Proof of Remark 2.8. We first work in one dimension. We use another property of $\mathcal{W}_{\varphi_{2}, \varphi_{1}}$.

(4) For each $\omega \in \mathbb{S}^{1}$, let $\sigma_{\omega}: \mathbb{R}^{2} \rightarrow \mathbb{R}^{2}$ be the rotation that maps $(1,0) \mapsto \omega$; then for all $z \in \mathbb{R}^{2}$ we have $\mathcal{W}_{\varphi_{2}, \varphi_{1}}\left(\sigma_{\omega} z\right)=\mathcal{W}_{\mathcal{F} \omega_{\varphi_{2}}, F \omega_{\varphi_{1}}}(z)$.

In others words, the fractional Fourier transform is the metaplectic operator corresponding to rotation. For example, in [9] see Proposition (1.94)(c) for the $\omega=(1,0)$ case (the usual Fourier transform) and Chapter 4 for discussion of metaplectic operators (especially Proposition (4.28) for the relationship to the Wigner transform). For more information on the fractional Fourier transform, see for example [15].

Combining property 4 with property 2 we obtain

$$
\int_{\left\{z^{\prime} \in \mathbb{R}^{2}: z^{\prime} \cdot \omega=\lambda\right\}} \mathcal{W}_{\varphi_{2}, \varphi_{1}}\left(z^{\prime}\right) \mu_{1}\left(\mathrm{~d} z^{\prime}\right)=\mathcal{F}^{\omega} \varphi_{2}(\lambda) \overline{\mathcal{F}^{\omega} \varphi_{1}(\lambda)} .
$$

(This is sometimes call the Radon-Wigner transform, since it is the Radon transform of the Wigner distribution.) But $Q_{\omega}$ is the antiderivative of this expression, so Remark 2.8 is immediate from this. For higher dimensions, we apply similar reasoning component-wise, so that $T_{\omega}$ is the composition of component-wise fractional Fourier transform operators. 


\section{Proof}

3.1. Overview. There are two steps to the proof of Theorem 2.4, which are distilled into the two lemmas in this subsection.

To avoid dealing with the scaling parameter $r$ throughout the whole proof, we will give names to the rescaled versions of $a$ and $\Omega$. We write

$$
T_{r}\left[a \chi_{\Omega}\right]=\operatorname{op}\left[W *\left(b \chi_{\Sigma}\right)\right], \quad \text { where } b:=a(\cdot / r), \Sigma:=r \Omega .
$$

The two lemmas will be proved in terms of general $b, \Sigma$ without explicit reference to the fact that they are rescaled versions of other objects. However, in each lemma the remainder scales in such a way that it is $O\left(r^{2 d-2}\right)$ when $b$ and $\Sigma$ are of this form.

The first step is composition, where we find an approximation of the Weyl symbol of $f\left(T_{r}\left[a \chi_{\Omega}\right]\right)$.

Lemma 3.1. Let $W, b, \Sigma, f$ satisfy Condition 2.1 and Condition 2.3, and let $\partial \Sigma$ have tubular radius of at least 1 . Then there exists $R$ such that

$$
\left\|f\left(\mathrm{op}\left[W *\left(b \chi_{\Sigma}\right)\right]\right)-\mathrm{op}\left[f\left(W *\left(b \chi_{\Sigma}\right)\right)\right]\right\|_{1} \leqslant R(b, \Sigma ; W, f),
$$

where $R$ satisfies the scaling property

$$
R(b, \Sigma ; W, f)=r^{2 d-2} R(a, \Omega ; W, f), \quad \text { for } b=a(\cdot / r), \Sigma=r \Omega .
$$

This is proved in §3.2. First, in Lemma 3.4, we will prove a trace norm bound for the composition of general Weyl operators. Next, in Lemma 3.6, we will apply this to the operator op $[W *(b \chi \Sigma)]$. A naive application would result in a trace norm bound that includes the four terms

$$
\begin{array}{cc}
\|\nabla b\|_{L^{\infty}(\Sigma)}\|\nabla b\|_{L^{1}(\Sigma)}, & \|\nabla b\|_{L^{\infty}(\Sigma)}\|b\|_{L^{1}(\partial \Sigma)}, \\
\|b\|_{L^{1}(\partial \Sigma)}\|\nabla b\|_{L^{\infty}(\Sigma)}, & \|b\|_{L^{\infty}(\partial \Sigma)}\|b\|_{L^{1}(\partial \Sigma)} .
\end{array}
$$

The first three terms are $O\left(r^{2 d-2}\right)$ as required, but the final one is $O\left(r^{2 d-1}\right)$. The proof of Lemma 3.6 involves some delicate cancellation using the geometry of $\partial \Sigma$ to obtain a better bound. This completes the proof of Lemma 3.1 in the case that $f(t)=t^{2}$; at the end of $\$ 3.2$ the proof is given for general $f$. 
Combined with the fact that $|\operatorname{tr} A| \leqslant\|A\|_{1}$ for every trace class operator $A$, Lemma 3.1 tells us that

$$
\operatorname{tr} f\left(T_{r}\left[a \chi_{\Omega}\right]\right)=\operatorname{trop}\left[f\left(W *\left(b \chi_{\Sigma}\right)\right)\right]+O\left(r^{2 d-2}\right) .
$$

The trace is given by the integral of the Weyl symbol (see \$2.1). The proof of Theorem 2.4 is thus completed by finding the asymptotics of this integral, which is done in the following lemma.

Lemma 3.2. Let $W, b, \Sigma, f$ satisfy Condition 2.1 and Condition 2.3. Then there exists $R$ such that, in the notation of Theorem 2.4, we have

$$
\left|\int_{\mathbb{R}^{2 d}} f\left(W *\left(b \chi_{\Sigma}\right)(z)\right) \mathrm{d} z-\left(A_{0}(b, \Sigma, f)+A_{1}(b, \Sigma, f)\right)\right| \leqslant R(b, \Sigma ; W, f),
$$

where $R$ satisfies the scaling property

$$
R(b, \Sigma ; W, f)=r^{2 d-2} R(a, \Omega ; W, f), \quad \text { for } b=a(\cdot / r), \Sigma=r \Omega .
$$

This is proved in $\S 3.3$. The proof begins by noting that, because the integral of $W$ is 1 and $f(0)=0$, the integral of $f\left(W *\left(b \chi_{\Sigma}\right)(z)\right)$ equals

$$
\int_{\Sigma} f(b(z)) \mathrm{d} z+\int_{\mathbb{R}^{2 d}}\left(f\left(W *\left(\chi_{\Sigma} b\right)(z)\right)-W *\left(\chi_{\Sigma} f(b)\right)(z)\right) \mathrm{d} z .
$$

The first term is simply $A_{0}(b, \Sigma, f)$, which equals $r^{2 d} A_{0}(a, \Omega, f)$. The second term is very similar to $A_{1}(b, \Sigma, f)$; in particular its integrand is concentrated near to $\partial \Sigma$. However, unlike $A_{1}$, it is not of the correct asymptotic form; that is, it does not equal $r^{2 d-1}$ multiplied by its unscaled version. The proof proceeds by using the local geometry of $\partial \Sigma$ to show that this integral is indeed approximately equal to $A_{1}$.

3.2. Step 1: Composition. In this subsection we prove Lemma 3.1, proceeding as discussed in $\$ 3.1$.

Notation 3.3. In this subsection we frequently decompose vectors $z \in \mathbb{R}^{2 d}$ as $z=\left(z_{1}, z_{2}\right)$, where $z_{1}, z_{2} \in \mathbb{R}^{d}$. Furthermore, we use the notation

$$
\langle\boldsymbol{x}\rangle:=\left(1+|\boldsymbol{x}|^{2}\right)^{1 / 2} .
$$

We start by proving trace norm and operator norm bounds for the error in replacing the Weyl symbol of composition by a finite number of terms in the series expansion. In fact we only need the trace norm bound, and only for $n=0$, but the full result is no harder to prove and the author hopes that it may be of general interest. 
Lemma 3.4. Let $p, q$ be infinitely differentiable functions on $\mathbb{R}^{2 d}$ such that $\partial^{\alpha} p$, $\partial^{\alpha} q \in L^{1}\left(\mathbb{R}^{2 d}\right)$ for each $\alpha \in \mathbb{N}_{0}^{2 d}$. Let $n \in \mathbb{N}_{0}, G \in \mathbb{N}_{0}$. Set

$$
F_{j}(\boldsymbol{x}, \boldsymbol{y}):=\frac{\mathrm{i}^{j}}{j ! 2^{j}}\left(\nabla_{\boldsymbol{x}_{1}} \cdot \nabla_{\boldsymbol{y}_{2}}-\nabla_{\boldsymbol{x}_{2}} \cdot \nabla_{\boldsymbol{y}_{1}}\right)^{j}(p(\boldsymbol{x}) q(\boldsymbol{y})), \quad c_{n}(\boldsymbol{z}):=\sum_{j=0}^{n} F_{j}(\boldsymbol{z}, \boldsymbol{z}) .
$$

Then

$$
\begin{gathered}
\left\|\mathrm{op}[p] \mathrm{op}[q]-\mathrm{op}\left[c_{n}\right]\right\|_{1} \leqslant C_{d, G} \sum_{\substack{\alpha \in \mathbb{N}_{0}^{4 d} \\
|\alpha| \leqslant G+4 d+2}} \int_{\mathbb{R}^{2 d}} \int_{\mathbb{R}^{2 d}} \frac{\left|\partial^{\alpha} F_{n+1}(\boldsymbol{x}, \boldsymbol{y})\right|}{\langle\boldsymbol{x}-\boldsymbol{y}\rangle^{G}} \mathrm{~d} \boldsymbol{x} \mathrm{d} \boldsymbol{y}, \\
\left\|\mathrm{op}[p] \mathrm{op}[q]-\mathrm{op}\left[c_{n}\right]\right\| \leqslant C_{d, G}^{\prime} \sum_{\substack{\alpha \in \mathbb{N}_{0}^{4} d \\
|\alpha| \leqslant G+3 d+3}} \int_{\mathbb{R}^{2 d}}\left(\sup _{\substack{\boldsymbol{x}, \boldsymbol{y} \in \mathbb{R}^{2 d} \\
\boldsymbol{x}-\boldsymbol{y}=\boldsymbol{v}}} \frac{\left|\partial^{\alpha} F_{n+1}(\boldsymbol{x}, \boldsymbol{y})\right|}{\langle\boldsymbol{x}-\boldsymbol{y}\rangle^{G}}\right) \mathrm{d} \boldsymbol{v} .
\end{gathered}
$$

The constants $C_{d, G}$ and $C_{d, G}^{\prime}$ depend only on $d$ and $G$ (not $n$ ).

The proof contains ideas used in the usual Weyl calculus adapted for use in these norm bounds. However, care has been taken to explicitly express the estimate in terms of the symbol rather than symbol class seminorms, and to preserve the cancellation between the terms within $F_{n+1}$. See the remark following the proof for a more detailed comparison.

Proof. It suffices to prove the result for $p, q \in \mathcal{S}\left(\mathbb{R}^{2 d}\right)$. Let $p \# q$ denote the Weyl symbol of op $[p]$ op $[q]$. We have $[9,(2.44 \mathrm{~b})]$

$$
p \# q(z)=\frac{1}{\pi^{2 d}} \int_{\mathbb{R}^{2 d}} \int_{\mathbb{R}^{2 d}} p(z-\boldsymbol{x}) q(\boldsymbol{z}-\boldsymbol{y}) \mathrm{e}^{2 \mathrm{i} \sigma(\boldsymbol{x}, \boldsymbol{y})} \mathrm{d} \boldsymbol{y} \mathrm{d} \boldsymbol{x},
$$

sometimes called the twisted product or Moyal product of $p$ and $q$, where

$$
\sigma(x, y):=x_{1} \cdot y_{2}-y_{1} \cdot x_{2} .
$$

We apply Taylor's theorem to $p$. The corresponding term of $p \# q(z)$ is

$$
T_{j}(z):=\frac{1}{\pi^{2 d}} \int_{\mathbb{R}^{2 d}} \int_{\mathbb{R}^{2 d}} \frac{1}{j !}\left(-\boldsymbol{x} \cdot \nabla_{p}\right)^{j} p(z) q(\boldsymbol{z}-\boldsymbol{y}) \mathrm{e}^{2 \mathrm{i} \sigma(\boldsymbol{x}, \boldsymbol{y})} \mathrm{d} \boldsymbol{y} \mathrm{d} \boldsymbol{x},
$$

where $\nabla_{p}$ indicates that the gradient is being taken only of $p$. Denote

$$
\tilde{\nabla}_{\boldsymbol{y}}:=\left(\nabla_{\boldsymbol{y}_{2}},-\nabla_{\boldsymbol{y}_{1}}\right)
$$

so that $2 \mathrm{i} x \mathrm{e}^{2 \mathrm{i} \sigma(\boldsymbol{x}, \boldsymbol{y})}=\widetilde{\nabla}_{\boldsymbol{y}} \mathrm{e}^{2 \mathrm{i} \sigma(\boldsymbol{x}, \boldsymbol{y})}$; then integrating by parts gives

$$
T_{j}(z)=\frac{1}{\pi^{2 d}} \int_{\mathbb{R}^{2 d}} \int_{\mathbb{R}^{2 d}} \frac{(-1)^{j}}{j !(2 \mathrm{i})^{j}}\left(\nabla_{p} \cdot \widetilde{\nabla}_{q}\right)^{j} p(z) q(\boldsymbol{z}-\boldsymbol{y}) \mathrm{e}^{2 \mathrm{i} \sigma(\boldsymbol{x}, \boldsymbol{y})} \mathrm{d} \boldsymbol{y} \mathrm{d} \boldsymbol{x} .
$$


By the Fourier inversion theorem we conclude that

$$
T_{j}(z)=\frac{(-1)^{j}}{j !(2 \mathrm{i})^{j}}\left(\nabla_{p} \cdot \tilde{\nabla}_{q}\right)^{j} p(z) q(z)=F_{j}(z, z) .
$$

Denote the symbol of the remainder by

$$
R_{n+1}(z):=a \# b(z)-c_{n}(z),
$$

which equals

$\frac{1}{\pi^{2 d}} \int_{\mathbb{R}^{2 d}} \int_{\mathbb{R}^{2 d}} \int_{0}^{1} \frac{1}{n !}(1-t)^{n}\left(-\boldsymbol{x} \cdot \nabla_{p}\right)^{n+1} p(z-t \boldsymbol{x}) q(\boldsymbol{z}-\boldsymbol{y}) \mathrm{e}^{2 \mathrm{i} \sigma(\boldsymbol{x}, \boldsymbol{y})} \mathrm{d} t \mathrm{~d} \boldsymbol{y} \mathrm{d} \boldsymbol{x}$.

Integrating by parts in the same way as the other terms, we find that $R_{n+1}(z)=\frac{1}{\pi^{2 d}} \int_{0}^{1} \int_{\mathbb{R}^{2 d}} \int_{\mathbb{R}^{2 d}}(n+1)(1-t)^{n} F_{n+1}(z-t \boldsymbol{x}, \boldsymbol{z}-\boldsymbol{y}) \mathrm{e}^{2 \mathrm{i} \sigma(\boldsymbol{x}, \boldsymbol{y})} \mathrm{d} \boldsymbol{y} \mathrm{d} \boldsymbol{x} \mathrm{d} t$.

Change variables $t \boldsymbol{x}=t \boldsymbol{u}+\frac{1}{2} \boldsymbol{v}$ and $\boldsymbol{y}=t \boldsymbol{u}-\frac{1}{2} \boldsymbol{v}$. This has Jacobian 1 and satisfies $\sigma(\boldsymbol{x}, \boldsymbol{y})=\sigma(\boldsymbol{v}, \boldsymbol{u})$, so

$$
R_{n+1}(z)=\frac{1}{\pi^{2 d}} \int_{0}^{1} \int_{\mathbb{R}^{2 d}} \int_{\mathbb{R}^{2 d}} f_{n+1}(z, \boldsymbol{u}, \boldsymbol{v} ; t) \mathrm{e}^{2 \mathrm{i} \sigma(\boldsymbol{v}, \boldsymbol{u})} \mathrm{d} \boldsymbol{u} \mathrm{d} \boldsymbol{v} \mathrm{d} t,
$$

where

$$
f_{n+1}(\boldsymbol{z}, \boldsymbol{u}, \boldsymbol{v} ; t):=(n+1)(1-t)^{n} F_{n+1}\left(z-\left(t \boldsymbol{u}+\frac{1}{2} \boldsymbol{v}\right), \boldsymbol{z}-\left(t \boldsymbol{u}-\frac{1}{2} \boldsymbol{v}\right)\right) .
$$

Define the operator

$$
P_{x, y}:=\frac{1+\frac{1}{2} \mathrm{i} \boldsymbol{y} \cdot \tilde{\nabla}_{\boldsymbol{x}}}{1+|\boldsymbol{y}|^{2}} \Longrightarrow P_{\boldsymbol{x}, \boldsymbol{y}}^{\mathrm{T}}=\frac{1-\frac{1}{2} \mathrm{i} \boldsymbol{y} \cdot \tilde{\nabla}_{\boldsymbol{x}}}{1+|\boldsymbol{y}|^{2}}
$$

so that $P_{\boldsymbol{v}, \boldsymbol{u}} \mathrm{e}^{\mathrm{2} \mathrm{i} \sigma(\boldsymbol{v}, \boldsymbol{u})}=\mathrm{e}^{2 \mathrm{i} \sigma(\boldsymbol{v}, \boldsymbol{u})}$ and $P_{\boldsymbol{u}, \boldsymbol{v}}^{\mathrm{T}} \mathrm{e}^{2 \mathrm{i} \sigma(\boldsymbol{v}, \boldsymbol{u})}=\mathrm{e}^{\mathrm{2} \mathrm{i} \sigma(\boldsymbol{v}, \boldsymbol{u})}$, and so and so the remainder $R_{n+1}(z)$ equals

$$
\frac{1}{\pi^{2 d}} \int_{0}^{1} \int_{\mathbb{R}^{2 d}} \int_{\mathbb{R}^{2 d}}\left(\left(P_{\boldsymbol{u}, \boldsymbol{v}}\right)^{M}\left(P_{\boldsymbol{v}, \boldsymbol{u}}^{\mathrm{T}}\right)^{L} f_{n+1}(\boldsymbol{z}, \boldsymbol{u}, \boldsymbol{v} ; t)\right) \mathrm{e}^{2 \mathrm{i} \sigma(\boldsymbol{v}, \boldsymbol{u})} \mathrm{d} \boldsymbol{u} \mathrm{d} \boldsymbol{v} \mathrm{d} t .
$$


For the interactions between $P_{\boldsymbol{u}, \boldsymbol{v}}$ and $P_{\boldsymbol{v}, \boldsymbol{u}}^{\mathrm{T}}$ we use the fact that for all $|\beta| \leqslant L$ and $|\gamma| \leqslant M$ we have

$$
\left|\partial_{\boldsymbol{u}}^{\gamma}\left(\frac{\boldsymbol{u}^{\beta}}{\langle\boldsymbol{u}\rangle^{2 L}}\right)\right| \leqslant C_{L, M} \frac{1}{\langle\boldsymbol{u}\rangle^{L}},
$$

for some constant $C_{L, M}$; thus $\left|\partial^{\alpha} R_{n+1}(z)\right|$ is bounded by a constant multiple of

$$
\sum_{|\beta| \leqslant L} \sum_{|\gamma| \leqslant M} \int_{0}^{1} \int_{\mathbb{R}^{2 d}} \int_{\mathbb{R}^{2 d}} \frac{\left|\partial_{\boldsymbol{z}}^{\alpha} \partial_{\boldsymbol{u}}^{\gamma} \partial_{\boldsymbol{v}}^{\beta} f_{n+1}(\boldsymbol{z}, \boldsymbol{u}, \boldsymbol{v} ; t)\right|}{\langle\boldsymbol{u}\rangle^{L}\langle\boldsymbol{v}\rangle^{M}} \mathrm{~d} \boldsymbol{u} \mathrm{d} \boldsymbol{v} \mathrm{d} t .
$$

Now choose $L=2 d+1, M=G$ and use the trace norm and operator norm bounds for Weyl operators (see \$2.1). Translating $z^{\prime}:=\boldsymbol{z}-\boldsymbol{t} \boldsymbol{u}$ and evaluating the $\mathrm{d} t$ integral (which cancels with $n+1$ ) gives the stated result.

Remark 3.5. We compare the above lemma with the usual symbolic calculus for Weyl operators. The decay in the integrand of $R_{n}$ could have been obtained without changing variables from $(\boldsymbol{x}, \boldsymbol{y})$ to $(\boldsymbol{u}, \boldsymbol{v})$; this bounds $\left|\partial^{\alpha} R_{n}(z)\right|$ by a constant multiple of

$$
\sum_{|\beta| \leqslant L} \sum_{|\gamma| \leqslant M} \int_{0}^{1} \int_{\mathbb{R}^{2 d}} \int_{\mathbb{R}^{2 d}} \frac{\left|\partial_{z}^{\alpha} \partial_{\boldsymbol{x}}^{\gamma} \partial_{\boldsymbol{y}}^{\beta} \tilde{f}_{n+1}(\boldsymbol{z}, \boldsymbol{x}, \boldsymbol{y} ; t)\right|}{\langle\boldsymbol{x}\rangle^{L}\langle\boldsymbol{y}\rangle^{M}} \mathrm{~d} \boldsymbol{x} \mathrm{d} \boldsymbol{y} \mathrm{d} t,
$$

where

$$
\tilde{f}_{n+1}(\boldsymbol{z}, \boldsymbol{x}, \boldsymbol{y} ; t):=(n+1)(1-t)^{n} F_{n+1}(\boldsymbol{z}-\sqrt{t} \boldsymbol{x}, \boldsymbol{z}-\sqrt{t} \boldsymbol{y}) .
$$

Using the notation of [9, Chapter 2], this is the bound used to show that if $p \in S_{\rho, 0}^{m_{1}}$, $q \in S_{\rho, 0}^{m_{2}}$ then $p \# q-c_{n} \in S_{\rho, 0}^{m_{1}+m_{2}-\rho(n+1)}$, see [9, Theorem (2.49)], although it is not computed explicitly there. This only gives the result when $\delta=0$; the general case could be handled in a similar way, but derivatives in $x_{1}$ and $x_{2}$ etc. would need to be tracked separately.

The next lemma, in combination with the previous one, proves Lemma 3.1 in the special case that $f(t)=t^{2}$, and contains the essential idea of the general case. To simplify its statement and use, introduce the notation

$$
\mathrm{M}^{G, D}(F):=\sum_{\substack{\alpha \in \mathbb{N}_{0}^{4 d} \\|\alpha| \leqslant D}} \int_{\mathbb{R}^{2 d}} \int_{\mathbb{R}^{2 d}} \frac{\left|\partial^{\alpha} F(\boldsymbol{x}, \boldsymbol{y})\right|}{\langle\boldsymbol{x}-\boldsymbol{y}\rangle^{G}} \mathrm{~d} \boldsymbol{x} \mathrm{d} \boldsymbol{y} .
$$


In particular, the trace norm bound in Lemma 3.4 is a constant multiple of $\mathrm{M}^{G, G+4 d+2}\left(F_{n}\right)$.

Lemma 3.6. Let $W, b, \Sigma$ satisfy Condition 2.1 and let the boundary of $\Sigma$ satisfy $\tau(\partial \Sigma) \geqslant 1$ (see 4 ). Set

$$
F(\boldsymbol{x}, \boldsymbol{y}):=\left(\nabla_{\boldsymbol{x}_{1}} \cdot \nabla_{\boldsymbol{y}_{2}}-\nabla_{\boldsymbol{x}_{2}} \cdot \nabla_{\boldsymbol{y}_{1}}\right)\left(W *\left(b \chi_{\Sigma}\right)(\boldsymbol{x}) W *\left(b \chi_{\Sigma}\right)(\boldsymbol{y})\right) .
$$

Set $G:=2 d+2, D:=6 d+4$. Then

$$
\mathrm{M}^{G, D}(F) \lesssim\|\nabla b\|_{L^{\infty}(\Sigma)}\left(\|\nabla b\|_{L^{1}(\Sigma)}+\|b\|_{L^{1}(\partial \Sigma)}\right)+\frac{\|b\|_{L^{\infty}(\partial \Sigma)}\|b\|_{L^{1}(\partial \Sigma)}}{\tau(\partial \Sigma)} .
$$

(Recall from $\S 2.1$ that the constant implicit in $\lesssim$ depends on $W$, but not on $b$ or $\Sigma$.)

Proof. We have

$$
F(\boldsymbol{x}, \boldsymbol{y})=\sum_{j=1}^{d}\left(\partial_{\left(\boldsymbol{x}_{1}\right)_{j}} \partial_{\left(\boldsymbol{y}_{2}\right)_{j}}-\partial_{\left(\boldsymbol{x}_{2}\right)_{j}} \partial_{\left(\boldsymbol{y}_{1}\right)_{j}}\right)\left(W *\left(b \chi_{\Sigma}\right)(\boldsymbol{x}) W *\left(b \chi_{\Sigma}\right)(\boldsymbol{y})\right) .
$$

For $j \in\{1, \ldots, d\}$ we have

$$
\partial_{\left(z_{1}\right)_{j}} W *\left(b \chi_{\Sigma}\right)(z)=g_{1, j}(z)+h_{1, j}(z),
$$

where

$$
g_{1, j}(\boldsymbol{z}):=\int_{\partial \Sigma} W\left(z-\boldsymbol{z}^{\prime}\right) b\left(\boldsymbol{z}^{\prime}\right)\left(\boldsymbol{n}_{1}\right)_{j}\left(\boldsymbol{z}^{\prime}\right) \mathrm{d} \boldsymbol{z}^{\prime}, \quad h_{1, j}(\boldsymbol{z}):=W *\left(\chi_{\Sigma} \partial_{\left(z_{1}\right)_{j}} b\right)(z),
$$

and similarly for $\partial_{\left(z_{2}\right)_{j}} W *\left(b \chi_{\Sigma}\right)(z)$. Thus, using the symmetry and subadditivity of $\mathrm{M}(\cdot)$, we have

$$
\begin{aligned}
\mathrm{M}^{G, D}(F) \leqslant \sum_{j=1}^{d}( & \mathrm{M}^{G, D}\left(g_{1, j}(\boldsymbol{x}) g_{2, j}(\boldsymbol{y})-g_{2, j}(\boldsymbol{x}) g_{1, j}(\boldsymbol{y})\right) \\
& +2 \mathrm{M}^{G, D}\left(g_{1, j}(\boldsymbol{x}) h_{2, j}(\boldsymbol{y})\right)+2 \mathrm{M}^{G, D}\left(g_{2, j}(\boldsymbol{x}) h_{1, j}(\boldsymbol{y})\right) \\
& \left.+2 \mathrm{M}^{G, D}\left(h_{1, j}(\boldsymbol{x}) h_{2, j}(\boldsymbol{y})\right)\right) .
\end{aligned}
$$

It is easy to check that

$$
\begin{aligned}
\mathrm{M}^{G, D}\left(g_{1, j}(\boldsymbol{x}) h_{2, j}(\boldsymbol{y})\right) & \lesssim\|b\|_{L^{1}(\partial \Sigma)}\|\nabla b\|_{L^{\infty}(\Sigma)}, \\
\mathrm{M}^{G, D}\left(g_{2, j}(\boldsymbol{x}) h_{1, j}(\boldsymbol{y})\right) & \lesssim\|b\|_{L^{1}(\partial \Sigma)}\|\nabla b\|_{L^{\infty}(\Sigma)}, \\
\mathrm{M}^{G, D}\left(h_{1, j}(\boldsymbol{x}) h_{2, j}(\boldsymbol{y})\right) & \lesssim\|\nabla b\|_{L^{1}(\Sigma)}\|\nabla b\|_{L^{\infty}(\Sigma)} .
\end{aligned}
$$


It remains to bound the first term. First note that

$$
\begin{aligned}
g_{1, j}(\boldsymbol{x}) g_{2, j}(\boldsymbol{y}) & -g_{2, j}(\boldsymbol{x}) g_{1, j}(\boldsymbol{y}) \\
& =\int_{\partial \Sigma} \int_{\partial \Sigma} W\left(\boldsymbol{x}-\boldsymbol{x}^{\prime}\right) b\left(\boldsymbol{x}^{\prime}\right) W\left(\boldsymbol{y}-\boldsymbol{y}^{\prime}\right) b\left(\boldsymbol{y}^{\prime}\right) \boldsymbol{m}\left(\boldsymbol{x}^{\prime}, \boldsymbol{y}^{\prime}\right) \mathrm{d} \boldsymbol{x}^{\prime} \mathrm{d} \boldsymbol{y}^{\prime},
\end{aligned}
$$

where for each $\boldsymbol{x}^{\prime}, \boldsymbol{y}^{\prime} \in \partial \Sigma$ we set

$$
\begin{aligned}
\boldsymbol{m}\left(\boldsymbol{x}^{\prime}, \boldsymbol{y}^{\prime}\right):= & \left(\boldsymbol{n}_{1}\right)_{j}\left(\boldsymbol{x}^{\prime}\right)\left(\boldsymbol{n}_{2}\right)_{j}\left(\boldsymbol{y}^{\prime}\right)-\left(\boldsymbol{n}_{2}\right)_{j}\left(\boldsymbol{x}^{\prime}\right)\left(\boldsymbol{n}_{1}\right)_{j}\left(\boldsymbol{y}^{\prime}\right) \\
= & \left(\left(\boldsymbol{n}_{1}\right)_{j}\left(\boldsymbol{x}^{\prime}\right)-\left(\boldsymbol{n}_{1}\right)_{j}\left(\boldsymbol{y}^{\prime}\right)\right)\left(\boldsymbol{n}_{2}\right)_{j}\left(\boldsymbol{y}^{\prime}\right) \\
& +\left(\left(\boldsymbol{n}_{2}\right)_{j}\left(\boldsymbol{y}^{\prime}\right)-\left(\boldsymbol{n}_{2}\right)_{j}\left(\boldsymbol{x}^{\prime}\right)\right)\left(\boldsymbol{n}_{1}\right)_{j}\left(\boldsymbol{y}^{\prime}\right) .
\end{aligned}
$$

Let $\ell\left(\boldsymbol{x}^{\prime}, \boldsymbol{y}^{\prime}\right)$ be the line segment connecting $\boldsymbol{x}^{\prime}$ to $\boldsymbol{y}^{\prime}$. When $\left|\boldsymbol{x}^{\prime}-\boldsymbol{y}^{\prime}\right| \leqslant \tau(\partial \Sigma) / 2$ we have $\ell\left(\boldsymbol{x}^{\prime}, \boldsymbol{y}^{\prime}\right) \subseteq \operatorname{tub}(\partial \Sigma, \tau(\partial \Sigma) / 2$ ) so by Lemma 4.12 (using the extension of $\boldsymbol{n}$ defined in $\S 4.1)$ we have

$$
\left|\left(n_{1}\right)_{j}\left(x^{\prime}\right)-\left(n_{1}\right)_{j}\left(y^{\prime}\right)\right| \leqslant\left|x^{\prime}-y^{\prime}\right| \sup _{z \in \ell\left(x^{\prime}, y^{\prime}\right)}|\nabla n(z)| \leqslant \frac{2\left|x^{\prime}-y^{\prime}\right|}{\tau(\partial \Sigma)} .
$$

When $\left|\boldsymbol{x}^{\prime}-\boldsymbol{y}^{\prime}\right| \geqslant \tau(\partial \Sigma) / 2$ we have

$$
\left|\left(\boldsymbol{n}_{1}\right)_{j}\left(\boldsymbol{x}^{\prime}\right)-\left(\boldsymbol{n}_{1}\right)_{j}\left(\boldsymbol{y}^{\prime}\right)\right| \leqslant 2 \leqslant \frac{4\left|\boldsymbol{x}^{\prime}-\boldsymbol{y}^{\prime}\right|}{\tau(\partial \Sigma)} .
$$

Similar bounds hold for $\boldsymbol{n}_{2}$, so

$$
\left|\boldsymbol{m}\left(\boldsymbol{x}^{\prime}, \boldsymbol{y}^{\prime}\right)\right| \leqslant \frac{8\left|\boldsymbol{x}^{\prime}-\boldsymbol{y}^{\prime}\right|}{\tau(\partial \Sigma)} \leqslant \frac{24\left\langle\boldsymbol{x}-\boldsymbol{x}^{\prime}\right\rangle\langle\boldsymbol{x}-\boldsymbol{y}\rangle\left\langle\boldsymbol{y}-\boldsymbol{y}^{\prime}\right\rangle}{\tau(\partial \Sigma)} .
$$

We also bound (using Lemma 4.13 with $U(z):=\langle z\rangle \partial^{\alpha} W(z)$ for the $\mathrm{d} \boldsymbol{x}^{\prime}$ integral)

$$
\begin{array}{r}
\int_{\partial \Sigma}\left\langle\boldsymbol{x}-\boldsymbol{x}^{\prime}\right\rangle\left|\partial^{\alpha} W\left(\boldsymbol{x}-\boldsymbol{x}^{\prime}\right) b\left(\boldsymbol{x}^{\prime}\right)\right| \mathrm{d} \boldsymbol{x}^{\prime} \\
\int_{\mathbb{R}^{2 d}} \int_{\partial \Sigma}\left\langle\boldsymbol{y}-\boldsymbol{y}^{\prime}\right\rangle\left|\partial^{\alpha} W\left(\boldsymbol{y}-\boldsymbol{y}^{\prime}\right) b\left(\boldsymbol{y}^{\prime}\right)\right| \mathrm{d} \boldsymbol{y}^{\prime} \mathrm{d} \boldsymbol{y} \lesssim\|b\|_{L^{1}(\partial \Sigma)} .
\end{array}
$$

We therefore obtain

$$
\mathrm{M}^{G, D}\left(g_{1, j}(\boldsymbol{x}) g_{2, j}(\boldsymbol{y})-g_{2, j}(\boldsymbol{x}) g_{1, j}(\boldsymbol{y})\right) \lesssim \frac{\|b\|_{L^{\infty}(\partial \Sigma)}\|b\|_{L^{1}(\partial \Sigma)}}{\tau(\partial \Sigma)} .
$$

Proof of Lemma 3.1. The way we complete this proof for general $f$ depends on the functional calculus in use; in other words, it depends on which of the two parts of Condition 2.3 is satisfied. In both cases we set $q:=W *\left(b \chi_{\Sigma}\right), G:=2 d+2$, $D:=6 d+4$, and

$$
F(\boldsymbol{x}, \boldsymbol{y}):=\left(\nabla_{\boldsymbol{x}_{1}} \cdot \nabla_{\boldsymbol{y}_{2}}-\nabla_{\boldsymbol{x}_{2}} \cdot \nabla_{\boldsymbol{y}_{1}}\right)(q(\boldsymbol{x}) q(\boldsymbol{y})) .
$$


Condition 2.3(1). For $j \geqslant 2$ by Lemma 3.4 we have

$$
\begin{aligned}
\left\|\mathrm{op}\left[q^{j+1}\right]-\mathrm{op}\left[q^{j}\right] \mathrm{op}[q]\right\|_{1} & \lesssim \mathrm{M}^{G, D}\left(\left(\nabla_{\boldsymbol{x}_{1}} \cdot \nabla_{\boldsymbol{y}_{2}}-\nabla_{\boldsymbol{x}_{2}} \cdot \nabla_{\boldsymbol{y}_{1}}\right)\left((q(\boldsymbol{x}))^{j} q(\boldsymbol{y})\right)\right) \\
& \leqslant(j-1)^{D}\left(c_{d, W}\|b\|_{L^{\infty}(\Sigma)}\right)^{j-1} \mathrm{M}^{G, D}(F),
\end{aligned}
$$

where $c_{d, W}$ is a constant. Summing from $j=1$ to $k-1$ and bounding the operator norm of op $[q]$ as in $\S 2.1$ we obtain

$$
\left\|(\mathrm{op}[q])^{k}-\mathrm{op}\left[q^{k}\right]\right\|_{1} \lesssim\left(1+(k-2)^{D+2}\right)\left(c_{d, W}\|b\|_{L^{\infty}(\Sigma)}\right)^{k-2} \mathrm{M}^{G, D}(F) .
$$

Thus

$$
\begin{aligned}
& \|f(\mathrm{op}[q])-\mathrm{op}[f(q)]\|_{1} \\
& \quad \leqslant \sum_{k=1}^{\infty}\left|f^{(k)}(0)\right|\left\|(\mathrm{op}[q])^{k}-\mathrm{op}\left[q^{k}\right]\right\|_{1} \\
& \quad \lesssim \mathrm{M}^{G, D}(F)\left(\sum_{k=2}^{\infty} \frac{\left|f^{(k)}(0)\right|}{k !}\left(1+(k-2)^{D+2}\right)\left(c_{d, W}\|b\|_{L^{\infty}(\Sigma)}\right)^{k-2}\right),
\end{aligned}
$$

which is convergent. Using Lemma 3.6 to bound $\mathrm{M}^{G, D}(F)$ gives the result.

Condition 2.3(2). We may assume that $f$ is compactly supported because only its values on a compact interval affect the meaning of $f(\mathrm{op}[q])$. It follows from the properties of the propagator $\mathrm{e}^{\mathrm{i} t} \mathrm{op}[q]$ that

$$
\begin{aligned}
& \|f(\mathrm{op}[q])-\mathrm{op}[f(q)]\|_{1} \\
& \quad \leqslant \frac{1}{\sqrt{2 \pi}} \int_{\mathbb{R}}\left(\int_{[0, t]}\left\|\mathrm{op}\left[\mathrm{e}^{\mathrm{i} s q}\right] \mathrm{op}[q]-\mathrm{op}\left[\mathrm{e}^{\mathrm{i} s q} q\right]\right\|_{1} \mathrm{~d} s\right)|\hat{f}(t)| \mathrm{d} t .
\end{aligned}
$$

(This may be seen by differentiating the operator $\mathrm{e}^{\mathrm{i} s \mathrm{op}[q]} \mathrm{op}\left[\mathrm{e}^{\mathrm{i} s q}\right]$ with respect to $s$ and integrating on $[0, t]$.) But by Lemma 3.4 we have

$$
\begin{aligned}
\| \mathrm{op} & {\left[\mathrm{e}^{\mathrm{i} s q}\right] \mathrm{op}[q]-\mathrm{op}\left[\mathrm{e}^{\mathrm{i} s q} q\right] \|_{1} } \\
& \lesssim \mathrm{M}^{G, D}\left(\left(\nabla_{\boldsymbol{x}_{1}} \cdot \nabla_{\boldsymbol{y}_{2}}-\nabla_{\boldsymbol{x}_{2}} \cdot \nabla_{\boldsymbol{y}_{1}}\right)\left(\mathrm{e}^{\mathrm{i} s q(\boldsymbol{x})} q(\boldsymbol{y})\right)\right) \\
& =\mathrm{M}^{G, D}\left(\mathrm{i} s \mathrm{e}^{\mathrm{i} s q(\boldsymbol{x})}\left(\nabla_{\boldsymbol{x}_{1}} \cdot \nabla_{\boldsymbol{y}_{2}}-\nabla_{\boldsymbol{x}_{2}} \cdot \nabla_{\boldsymbol{y}_{1}}\right)(q(\boldsymbol{x}) q(\boldsymbol{y}))\right) \\
& \lesssim\langle s\rangle^{D+1}\left\langle\|b\|_{L^{\infty}(\Sigma)}\right\rangle^{D} \mathrm{M}^{G, D}(F) .
\end{aligned}
$$

The result then follows from Lemma 3.6. 
3.3. Step 2: Trace asymptotics. In this subsection we prove Lemma 3.2. Set

$$
\begin{aligned}
I_{1} & :=\int_{\mathbb{R}^{2 d}}\left(f\left(W *\left(\chi_{\Sigma} b\right)(\boldsymbol{z})\right)-W *\left(\chi_{\Sigma} f(b)\right)(\boldsymbol{z})\right) \mathrm{d} \boldsymbol{z}, \\
I_{5} & :=\int_{\partial \Sigma} \int_{\mathbb{R}}\left(f\left(Q_{\boldsymbol{n}(\boldsymbol{u})}(\lambda) b(\boldsymbol{u})\right)-Q_{\boldsymbol{n}(\boldsymbol{u})}(\lambda) f(b(\boldsymbol{u}))\right) \mathrm{d} \lambda \mu_{2 d-1}(\mathrm{~d} \boldsymbol{u}) .
\end{aligned}
$$

As discussed in $\S 3.1$, we must show that when $b=a(\cdot / r)$ and $\Sigma=r \Omega$, we have $I_{1}=I_{5}+O\left(r^{2 d-2}\right)$.

Notation 3.7. In this subsection we will refer to the tubular radius of the boundary of $\Sigma$ very often, so instead of using the full notation $\tau(\partial \Sigma)$ (which for $\Sigma=r \Omega$ equals $r \tau(\partial \Omega))$ we will refer to it simply as $\tau$.

Proof of Lemma 3.2. Step 1. Restrict support of $f$. Depending on which part of Condition 2.3 is satisfied, either $f$ is a smooth function on $\mathbb{R}$ and $b, W$ are realvalued, or $f$ is a smooth function on $\mathbb{C}$. In both cases, $I_{1}$ and $I_{5}$ only depend on the value of $f(t)$ for

$$
|t| \leqslant\|b\|_{L^{\infty}(\Sigma)} \int_{\mathbb{R}^{2 d}}|W(z)| \mathrm{d} z,
$$

so we may restrict the support of $f$ to a compact set. In the remainder of the proof we refer to $\|f\|_{L^{\infty}}$ for the supremum of $|f|$ over that set, and similarly for $\left\|f^{\prime}\right\|_{L^{\infty}}$ and $\left\|f^{\prime \prime}\right\|_{L^{\infty}}$.

Step 2: Restrict support of $W$. Let $\widetilde{W}$ be the function defined for each $z \in \mathbb{R}^{2 d}$ by

$$
\tilde{W}(z):= \begin{cases}W(z)+K_{W, \tau} & \text { if }|z| \leqslant \frac{1}{2} \tau, \\ 0 & \text { if }|z|>\frac{1}{2} \tau,\end{cases}
$$

with $K_{W, \tau}$ chosen so that the integral of $\tilde{W}$ is 1 . The error in replacing $W$ by $\tilde{W}$ in $I_{1}$ and $I_{5}$ (including the reference to $W$ in the definition of $Q$ ) is bounded by

$$
\begin{aligned}
& 2\left\|f^{\prime}\right\|_{L^{\infty}}\|b\|_{L^{1}(\Sigma)} \int_{\mathbb{R}^{2 d}}|W(z)-\widetilde{W}(z)| \mathrm{d} z \\
& \quad+2\left\|f^{\prime}\right\|_{L^{\infty}}\|b\|_{L^{1}(\partial \Sigma)} \sup _{\omega \in \mathbb{S}^{2 d-1}} \int_{\mathbb{R}}\left|Q_{\omega}(\lambda)-\tilde{Q}_{\omega}(\lambda)\right| \mathrm{d} \lambda .
\end{aligned}
$$

Since $W \in \mathcal{S}\left(\mathbb{R}^{2 d}\right)$ these integrals can be bounded by any negative power of $\tau$; choosing to bound them by $1 / \tau^{2}$ and $1 / \tau$ respectively will suffice to satisfy the required scaling property. 
This will be useful later in the proof where certain integrals will be non-zero outside of a tubular neighbourhood of $\partial \Sigma$ so long as $W$ has sufficiently small compact support, and this will allow us to apply the results in $\S 4$. We also have, for each $k \in \mathbb{N}_{0}$,

$$
\int_{\mathbb{R}^{2 d}}\left(1+\left|z^{\prime}\right|\right)^{k}\left|\tilde{W}\left(z^{\prime}\right)\right| \mathrm{d} z^{\prime} \leqslant \int_{\mathbb{R}^{2 d}}\left(1+\left|z^{\prime}\right|\right)^{k}\left|W\left(z^{\prime}\right)\right| \mathrm{d} z^{\prime},
$$

so any bound depending on $\widetilde{W}$ in this way can be replaced by one depending on $W$ uniformly in $\tau$. For the rest of the proof we use $\widetilde{W}$ in place of $W$ without further comment.

$\underline{\text { Step 3: Extract } b \text { from convolution. Let }}$

$$
I_{2}:=\int_{\mathbb{R}^{2 d}}\left(f\left(W * \chi_{\Sigma}(z) b(z)\right)-W * \chi_{\Sigma}(z) f(b(z))\right) \mathrm{d} z .
$$

We will bound $\left|I_{1}-I_{2}\right|$. We can rewrite

$$
I_{1}-I_{2}=\int_{\mathbb{R}^{2 d}}\left(D_{1}(z)-D_{2}(z)\right) \mathrm{d} z,
$$

where

$$
\begin{aligned}
& D_{1}(z):=f\left(W *\left(\chi_{\Sigma} b\right)(z)\right)-f\left(W * \chi_{\Sigma}(z) b(z)\right), \\
& D_{2}(z):=W *\left(\chi_{\Sigma} f(b)\right)(z)-W * \chi_{\Sigma}(z) f(b(z)) .
\end{aligned}
$$

Using two terms of the Taylor expansion of $b$ and two terms of the Taylor expansion of $f$, we obtain

$$
\begin{aligned}
& \int_{\mathbb{R}^{2 d}}\left|D_{1}(z)-\left(-z^{\prime} W\left(z^{\prime}\right)\right) * \chi_{\Sigma}(z) \cdot \nabla b(z) f^{\prime}\left(W * \chi_{\Sigma}(z) b(z)\right)\right| \mathrm{d} z \\
& \quad \lesssim\left\|f^{\prime \prime}\right\|_{L^{\infty}}\|\nabla b\|_{L^{\infty}\left(\mathbb{R}^{2 d}\right)}\|\nabla b\|_{L^{1}\left(\mathbb{R}^{2 d}\right)}+\left\|f^{\prime}\right\|_{L^{\infty}}\|\nabla(\nabla b)\|_{L^{1}\left(\mathbb{R}^{2 d}\right)} .
\end{aligned}
$$

Using two terms of the Taylor expansion of $f(b)$, we obtain

$$
\begin{aligned}
& \int_{\mathbb{R}^{2 d}}\left|D_{2}(z)-\left(-z^{\prime} W\left(z^{\prime}\right)\right) * \chi_{\Sigma}(z) \cdot \nabla b(z) f^{\prime}(b(z))\right| \mathrm{d} z \\
& \quad \lesssim\left\|f^{\prime \prime}\right\|_{L^{\infty}}\|\nabla b\|_{L^{\infty}\left(\mathbb{R}^{2 d}\right)}\|\nabla b\|_{L^{1}\left(\mathbb{R}^{2 d}\right)}+\left\|f^{\prime}\right\|_{L^{\infty}}\|\nabla(\nabla b)\|_{L^{1}\left(\mathbb{R}^{2 d}\right)} .
\end{aligned}
$$


It thus remains to bound

$$
\int_{\mathbb{R}^{2 d}}\left|\left(z^{\prime} W\left(z^{\prime}\right)\right) * \chi_{\Sigma}(z) \cdot \nabla b(z)\left(f^{\prime}\left(W * \chi_{\Sigma}(z) b(z)\right)-f^{\prime}(b(z))\right)\right| \mathrm{d} z .
$$

This integral is zero outside of $\operatorname{tub}(\partial \Sigma, \tau / 2)$. Set $V\left(z^{\prime}\right):=\left(1+\left|z^{\prime}\right|\right) W\left(z^{\prime}\right)$. By Lemma 4.11 and Lemma 4.14, it is thus bounded by

$$
\begin{aligned}
& \left\|f^{\prime \prime}\right\|_{L^{\infty}} \int_{\operatorname{tub}(\partial \Sigma, \tau / 2)}\left|\left(z^{\prime} W\left(z^{\prime}\right)\right) * \chi_{\Sigma}(z) \cdot \nabla b(z) W * \chi_{\Sigma^{c}}(z) b(z)\right| \mathrm{d} z \\
& \quad \leqslant\left\|f^{\prime \prime}\right\|_{L^{\infty}}\|b\|_{L^{\infty}\left(\mathbb{R}^{2 d}\right)} \int_{\operatorname{tub}(\partial \Sigma, \tau / 2)} \mid V * \chi \Sigma(z) V * \chi_{\Sigma^{c}(z) \nabla b(z) \mid \mathrm{d} z} \\
& \quad \lesssim\left\|f^{\prime \prime}\right\|_{L^{\infty}\|b\|_{L^{\infty}\left(\mathbb{R}^{2 d}\right)}\left(\|\nabla b\|_{L^{1}(\partial \Sigma)}+\|\nabla(\nabla b)\|_{L^{1}\left(\mathbb{R}^{2 d}\right)}\right) .}
\end{aligned}
$$

Step 4: Approximate $b$ by its value on $\partial \Sigma$. Let

$$
I_{3}:=\int_{\operatorname{tub}(\partial \Sigma, \tau / 2)}\left(f\left(W * \chi_{\Sigma}(\boldsymbol{z}) b(\boldsymbol{u})\right)-W * \chi_{\Sigma}(\boldsymbol{z}) f(b(\boldsymbol{u}))\right) \mathrm{d} \boldsymbol{z},
$$

where for each $z \in \operatorname{tub}(\partial \Sigma, \tau)$ we define $\boldsymbol{u}:=\boldsymbol{z}-\delta(\boldsymbol{z}) \boldsymbol{n}(\boldsymbol{z}) \in \partial \Sigma$ (the signed distance function $\delta$ is defined in $\S 4.1$ ). The integrand of $I_{2}$ is zero outside of $z \in \operatorname{tub}(\partial \Sigma, \tau / 2)$, so by Lemma 4.11 (essentially Taylor's theorem on $b$ in the $\boldsymbol{n}(\boldsymbol{u})$ direction) and Lemma 4.14 we have

$$
\begin{aligned}
& \left|I_{2}-I_{3}\right| \\
& \quad \leqslant\left\|f^{\prime \prime}\right\|_{L^{\infty}}\|b\|_{L^{\infty}\left(\mathbb{R}^{2 d}\right)} \int_{\operatorname{tub}(\partial \Sigma, \tau / 2)}\left|b(\boldsymbol{z})-b(\boldsymbol{u})\|W * \chi \Sigma(z)\| W * \chi \Sigma^{c}(z)\right| \mathrm{d} \boldsymbol{z} \\
& \quad \lesssim\left\|f^{\prime \prime}\right\|_{L^{\infty}\|b\|_{L^{\infty}\left(\mathbb{R}^{2 d}\right)}\left(\|\nabla b\|_{L^{1}(\partial \Sigma)}+\|\nabla(\nabla b)\|_{L^{1}\left(\mathbb{R}^{2 d}\right)}\right) .}
\end{aligned}
$$

Step 5: Approximate $\Sigma$ locally by a half space. Let

$$
I_{4}:=\int_{\operatorname{tub}(\partial \Sigma, \tau / 2)}\left(f\left(Q_{\boldsymbol{n}(\boldsymbol{z})}(\delta(\boldsymbol{z})) b(\boldsymbol{u})\right)-Q_{\boldsymbol{n}(\boldsymbol{u})}(\delta(\boldsymbol{z})) f(b(\boldsymbol{u}))\right) \mathrm{d} \boldsymbol{z},
$$

where as before we define $\boldsymbol{u}:=\boldsymbol{z}-\delta(\boldsymbol{z}) \boldsymbol{n}(\boldsymbol{z}) \in \partial \Sigma$. By Lemma 4.8 and Lemma 4.10 we have

$$
\begin{aligned}
\mid I_{3} & -I_{4} \mid \\
& \lesssim\left\|f^{\prime}\right\|_{L^{\infty}} \int_{\partial \Sigma} \int_{-\tau / 2}^{\tau / 2}\left|b(\boldsymbol{u}) \| W * \chi \Sigma(\boldsymbol{u}+\lambda \boldsymbol{n}(\boldsymbol{u}))-Q_{\boldsymbol{n}(\boldsymbol{u})}(\lambda)\right| \mathrm{d} \lambda \mu_{2 d-1}(\mathrm{~d} \boldsymbol{u}) \\
& \leqslant\left\|f^{\prime}\right\|_{L^{\infty}}\|b\|_{L^{1}(\partial \Sigma)} \sup _{\boldsymbol{u} \in \partial \Omega} J(\boldsymbol{u}),
\end{aligned}
$$


where for each $\boldsymbol{u} \in \partial \Omega$ we set

$$
J(\boldsymbol{u}):=\int_{-\tau / 2}^{\tau / 2}\left|W * \chi_{\Sigma}(\boldsymbol{u}+\lambda \boldsymbol{n}(\boldsymbol{u}))-Q_{\boldsymbol{n}(\boldsymbol{u})}(\lambda)\right| \mathrm{d} \lambda .
$$

We will show that $J(\boldsymbol{u}) \lesssim 1 / \tau$. We have

$$
Q_{\boldsymbol{n}(\boldsymbol{u})}(\lambda)=W * \chi_{H}(\boldsymbol{u}+\lambda \boldsymbol{n}(\boldsymbol{u})), \quad H:=\left\{\boldsymbol{z}^{\prime} \in \mathbb{R}^{2 d}:\left(\boldsymbol{z}^{\prime}-\boldsymbol{u}\right) \cdot \boldsymbol{n}(\boldsymbol{u}) \geqslant 0\right\} .
$$

So, denoting symmetric difference by $\Delta$, we have

$$
\begin{aligned}
J(\boldsymbol{u}) & \leqslant \int_{-\tau / 2}^{\tau / 2}|W| * \chi_{\Sigma \Delta H}(\boldsymbol{u}+\lambda \boldsymbol{n}(\boldsymbol{u})) \mathrm{d} \lambda \\
& =\int_{-\tau / 2}^{\tau / 2} \int_{\Sigma \Delta H}\left|W\left(\boldsymbol{u}+\lambda \boldsymbol{n}(\boldsymbol{u})-\boldsymbol{z}^{\prime}\right)\right| \mathrm{d} \boldsymbol{z}^{\prime} \mathrm{d} \lambda .
\end{aligned}
$$

This integrand is non-zero only when $\left|\boldsymbol{u}+\lambda \boldsymbol{n}(\boldsymbol{u})-\boldsymbol{z}^{\prime}\right|<\tau / 2$ and $|\lambda|<\tau / 2$, so only when $\left|\boldsymbol{u}-\boldsymbol{z}^{\prime}\right|<\tau$. We may therefore use Remark 4.4 with $\boldsymbol{z}^{\prime}=\boldsymbol{u}+v_{\perp} \boldsymbol{n}(\boldsymbol{u})+\tilde{\boldsymbol{v}}$. This says that $z^{\prime} \in \Sigma \Delta H$ only when $\left|v_{\perp}\right| \leqslant|\tilde{\boldsymbol{v}}|^{2} / \tau$, so

$$
J(\boldsymbol{u}) \leqslant \int_{-\tau / 2}^{\tau / 2} \int_{\boldsymbol{n}(\boldsymbol{u}) \perp} \int_{-|\tilde{\boldsymbol{v}}|^{2} / \tau}^{|\tilde{\boldsymbol{v}}|^{2} / \tau}\left|W\left(\lambda \boldsymbol{n}(\boldsymbol{u})-v_{\perp} \boldsymbol{n}(\boldsymbol{u})-\tilde{\boldsymbol{v}}\right)\right| \mathrm{d} v_{\perp} \mu_{2 d-1}(\mathrm{~d} \tilde{\boldsymbol{v}}) \mathrm{d} \lambda .
$$

Translating $\lambda$ to $\eta:=\lambda-v_{\perp}$ and then setting $\boldsymbol{x}:=\eta \boldsymbol{n}(\boldsymbol{u})-\tilde{\boldsymbol{v}}$, we obtain

$$
\begin{aligned}
J(\boldsymbol{u}) & \leqslant \int_{\boldsymbol{n}(\boldsymbol{u})^{\perp}} \int_{-|\tilde{\boldsymbol{v}}|^{2} / \tau}^{|\tilde{\boldsymbol{v}}|^{2} / \tau} \int_{\mathbb{R}}|W(\eta \boldsymbol{n}(\boldsymbol{u})-\tilde{\boldsymbol{v}})| \mathrm{d} \eta \mathrm{d} v_{\perp} \mu_{2 d-1}(\mathrm{~d} \tilde{\boldsymbol{v}}) \\
& \leqslant \frac{2}{\tau} \int_{\mathbb{R}^{2 d}}|\boldsymbol{x}|^{2}|W(\boldsymbol{x})| \mathrm{d} \boldsymbol{x} \lesssim \frac{1}{\tau} .
\end{aligned}
$$

Step 6: Neglect Jacobian. By Lemma 4.8 we have

$$
I_{4}=\int_{\partial \Sigma} \int_{-\tau / 2}^{\tau / 2}\left(f\left(Q_{\boldsymbol{n}(\boldsymbol{z})}(\lambda) b(\boldsymbol{u})\right)-Q_{\boldsymbol{n}(\boldsymbol{u})}(\lambda) f(b(\boldsymbol{u}))\right) \operatorname{det}\left(I-\lambda S_{\boldsymbol{u}}\right) \mathrm{d} \lambda \mu_{2 d-1}(\mathrm{~d} \boldsymbol{u}) .
$$

In $I_{5}$ the integrand is zero except for when $-\tau / 2<\lambda<\tau / 2$, so using Lemma 4.10 to replace $\operatorname{det}\left(I-\lambda S_{\boldsymbol{u}}\right)$ with 1 , we have

$$
\begin{aligned}
\left|I_{4}-I_{5}\right| & \lesssim \frac{1}{\tau} \int_{\partial \Sigma} \int_{-\tau / 2}^{\tau / 2}|\lambda|\left|f\left(Q_{\boldsymbol{n}(\boldsymbol{z})}(\lambda) b(\boldsymbol{u})\right)-Q_{\boldsymbol{n}(\boldsymbol{u})}(\lambda) f(b(\boldsymbol{u}))\right| \mathrm{d} \lambda \mu_{2 d-1}(\mathrm{~d} \boldsymbol{u}) \\
& \leqslant \frac{2}{\tau}\left\|f^{\prime}\right\|_{L^{\infty}} \int_{\partial \Sigma}|b(\boldsymbol{u})| \mu_{2 d-1}(\mathrm{~d} \boldsymbol{u}) \int_{\mathbb{R}}|\lambda|\left|Q_{\boldsymbol{n}(\boldsymbol{z})}(\lambda)-\chi_{[0, \infty)}(\lambda)\right| \mathrm{d} \lambda \\
& \lesssim \frac{1}{\tau}\left\|f^{\prime}\right\|_{L^{\infty}}\|b\|_{L^{1}(\partial \Sigma)} .
\end{aligned}
$$




\section{Appendix: Tubular neighbourhood properties}

4.1. Definition and properties. Here we recall the definition of tubular neighbourhoods and some of their basic properties. Throughout this subsection let $\Omega \subseteq$ $\mathbb{R}^{m}$ be a closed set with $C^{2}$ boundary. In practice we will only need the results when $m$ is even, but everything applies equally to odd $m$. The material below is well known; see for example [10, Appendix; moved to \$14.6 in 1983 second edition] or [11].

Notation 4.1. Denote the inward normal vector field by $n: \partial \Omega \rightarrow \mathbb{R}^{m}$.

Definition 4.2. Let $t>0$. Define the open line segments

$$
\ell_{\mathrm{nor}}(\boldsymbol{u}, t, \partial \Omega):=\left\{\boldsymbol{u}+\lambda \boldsymbol{n}(\boldsymbol{u}) \in \mathbb{R}^{m}: \lambda \in(-t, t)\right\}
$$

and define the set

$$
\operatorname{tub}(\partial \Omega, t):=\bigcup_{\boldsymbol{u} \in \partial \Omega} \ell_{\text {nor }}(\boldsymbol{u}, t, \partial \Omega) .
$$

When the $\ell_{\text {nor }}(\boldsymbol{u}, t, \partial \Omega)$ are disjoint for all distinct $\boldsymbol{u} \in \partial \Omega$ we call tub $(\partial \Omega, t)$ a tubular neighbourhood of radius $t$.

For any $t>0$, the set tub $(\partial \Omega, t)$ is is precisely the set of points within distance $t$ of $\partial \Omega$. When $\Omega$ is compact, there always exists a $t>0$ such that $\partial \Omega$ has a tubular neighbourhood of radius $t$; this fact is called the tubular neighbourhood theorem. We denote maximum such radius that exists by $\tau(\partial \Omega$ ) (or set $\tau(\partial \Omega):=0$ if no such $t$ exists); it satisfies the scaling relationship, for $\lambda>0$,

$$
\tau(\lambda \partial \Omega)=\lambda \tau(\partial \Omega) .
$$

When $\tau(\partial \Omega)>0$ we write simply tub $(\partial \Omega)$ for the tube of this radius; that is,

$$
\operatorname{tub}(\partial \Omega):=\operatorname{tub}(\partial \Omega, \tau(\partial \Omega)) .
$$

Notation 4.3. For any $z \in \mathbb{R}^{m}$ and $t>0$, we denote the open ball in $\mathbb{R}^{m}$ centred on $z$ with radius $t$ by $B(z, t)$.

Remark 4.4. An equivalent condition to $\partial \Omega$ having a tubular neighbourhood of radius $t$ is that for each $\boldsymbol{u} \in \partial \Omega$ the balls $B(\boldsymbol{u} \pm \operatorname{tn}(\boldsymbol{u}), t)$ do not intersect $\partial \Omega$. This implies that locally the surface $\partial \Omega$ is approximately flat with uniform quadratic error. To state this explicitly, for $\boldsymbol{u} \in \partial \Omega, \boldsymbol{z} \in B(\boldsymbol{u}, \tau(\partial \Omega))$ set $\boldsymbol{v}:=\boldsymbol{z}-\boldsymbol{u}$, $v_{\perp}:=\boldsymbol{v} \cdot \boldsymbol{n}(\boldsymbol{u}), \tilde{\boldsymbol{v}}:=\boldsymbol{v}-v_{\perp} \boldsymbol{n}(\boldsymbol{u})$, so that $\boldsymbol{z}=\boldsymbol{u}+v_{\perp} \boldsymbol{n}(\boldsymbol{u})+\tilde{\boldsymbol{v}}$. (Then $\tilde{\boldsymbol{v}} \in \boldsymbol{n}(\boldsymbol{u})^{\perp}$ i.e. $\tilde{\boldsymbol{v}}$ is in the tangent space at $\boldsymbol{u}$.) Then

$$
\boldsymbol{z} \in \partial \Omega \Longrightarrow\left|v_{\perp}\right| \leqslant|\tilde{\boldsymbol{v}}|^{2} / \tau(\partial \Omega) .
$$


Definition 4.5. The signed distance function (also called the oriented distance function) is

$$
\delta_{\Omega}(z):=\left\{\begin{aligned}
\operatorname{dist}(z, \partial \Omega) & \text { if } z \in \Omega, \\
-\operatorname{dist}(z, \partial \Omega) & \text { if } z \notin \Omega .
\end{aligned}\right.
$$

Lemma 4.6. Let $\Omega$ have a boundary satisfying $\tau(\partial \Omega)>0$. Then $\delta_{\Omega}$ is twice continuously differentiable on $\operatorname{tub}(\partial \Omega)$. Further, let $\boldsymbol{z} \in \operatorname{tub}(\partial \Omega)$, and set $\boldsymbol{u} \in \partial \Omega$ to the (unique) nearest point to $z$ in $\partial \Omega$; then

$$
\nabla \delta_{\Omega}(z)=\nabla \delta_{\Omega}(\boldsymbol{u})=\boldsymbol{n}(\boldsymbol{u}), \quad \boldsymbol{z}=\boldsymbol{u}+\delta_{\Omega}(z) \boldsymbol{n}(\boldsymbol{u}) .
$$

Lemma 4.6 shows that $\nabla \delta_{\Omega}$ is a continuously differentiable extension of the inward normal vector field, so we write without ambiguity

$$
\boldsymbol{n}(z):=\nabla \delta_{\Omega}(z) \text { for all } z \in \operatorname{tub}(\partial \Omega) .
$$

In particular, $|\boldsymbol{n}(\boldsymbol{z})|=1$ and $(\boldsymbol{n}(\boldsymbol{z}) \cdot \nabla) \boldsymbol{n}(\boldsymbol{z})=\mathbf{0}$ for all $z \in \operatorname{tub}(\partial \Omega)$.

The primary use of tubular neighbourhoods in this article is to reparametrise points near to $\partial \Omega$ in terms of points on $\partial \Omega$ and the signed distance. To write the Jacobian for this we need to use the shape operator.

Definition 4.7. For each $\boldsymbol{u} \in \partial \Omega$, define the shape operator, also known as the Weingarten map, by

$$
S_{\boldsymbol{u}}:=\nabla \boldsymbol{n}(\boldsymbol{u})=\nabla\left(\nabla \delta_{\Omega}\right)(\boldsymbol{u}) .
$$

The associated quadratic form is called the second fundamental form.

The shape operator is usually defined as $\widetilde{S}_{\boldsymbol{u}}:=\nabla_{T_{\boldsymbol{u}} \partial \Omega} \boldsymbol{n}(\boldsymbol{u})$ (the gradient of the normal vector field in the tangent hyperplane), which is a square matrix of size $m-1$. However, because $(\boldsymbol{n}(\boldsymbol{u}) \cdot \nabla) \boldsymbol{n}(\boldsymbol{u})=\mathbf{0}$ we have $S_{\boldsymbol{u}}=\widetilde{S}_{\boldsymbol{u}} \oplus 0$, so the distinction will not affect what follows.

Since $S_{\boldsymbol{u}}$ is the Hessian of a real-valued function, it is a real symmetric matrix, and hence diagonalizable with real eigenvalues (called the principal curvatures). The operator norm of $S_{\boldsymbol{u}}$ equals its (absolutely) largest principal curvature and satisfies

$$
\left|S_{\boldsymbol{u}}\right| \leqslant \frac{1}{\tau(\partial \Omega)}
$$


Lemma 4.8. For any $0<t \leqslant \tau(\partial \Omega)$, the change of variables

$$
\begin{aligned}
\lambda:= & \delta_{\Omega}(z) \in(-t, t), \boldsymbol{u}:=\boldsymbol{z}-\delta_{\Omega}(z) \boldsymbol{n}(z) \in \partial \Omega \\
& \Longleftrightarrow \boldsymbol{z}=\boldsymbol{u}+\lambda \boldsymbol{n}(\boldsymbol{u}) \in \operatorname{tub}(\partial \Omega, t),
\end{aligned}
$$

has Jacobian $\operatorname{det}\left(I-\lambda S_{\boldsymbol{u}}\right)$. In other words, for any $f \in L^{1}(\operatorname{tub}(\partial \Omega, t))$ we have

$$
\int_{\operatorname{tub}(\partial \Omega, t)} f(z) \mathrm{d} z=\int_{\partial \Omega} \int_{(-t, t)} f(\boldsymbol{u}+\lambda \boldsymbol{n}(\boldsymbol{u})) \operatorname{det}\left(I-\lambda S_{\boldsymbol{u}}\right) \mathrm{d} \lambda \mu_{m-1}(\mathrm{~d} \boldsymbol{u}) .
$$

We will need one final fact, which will be used to bound the difference between nearby normals (Lemma 4.12).

Lemma 4.9. Let $\boldsymbol{z} \in \operatorname{tub}(\partial \Omega)$. Set $\boldsymbol{u} \in \partial \Omega$ to be the nearest point on $\partial \Omega$ to $\boldsymbol{z}$, and set $U$ to an orthogonal matrix that diagonalises $S_{\boldsymbol{u}}$ i.e.

$$
S_{\boldsymbol{u}}=U^{-1} \operatorname{diag}\left\{\kappa_{1}, \ldots, \kappa_{m-1}, 0\right\} U
$$

where $\kappa_{j}$ are the principal curvatures at $\boldsymbol{u}$. Then

$$
\nabla \boldsymbol{n}(z)=U^{-1} \operatorname{diag}\left\{\frac{-\kappa_{1}}{1-\delta_{\Omega}(z) \kappa_{1}}, \ldots, \frac{-\kappa_{m-1}}{1-\delta_{\Omega}(z) \kappa_{m-1}}, 0\right\} U .
$$

4.2. Some basic consequences. This subsection collects some simple consequences of the tubular neighbourhood theory described in $\S 4.1$, used in $\S 3$ to prove Theorem 2.4. We will first need a pair of simple bounds on the Jacobian in Lemma 4.8 .

Lemma 4.10. For all $|\lambda| \leqslant \tau(\partial \Omega) / 2$ and $\boldsymbol{u} \in \partial \Omega$ we have

$$
\begin{gathered}
\left(\frac{1}{2}\right)^{m-1} \leqslant \operatorname{det}\left(I-\lambda S_{\boldsymbol{u}}\right) \leqslant\left(\frac{3}{2}\right)^{m-1}, \\
\left|\operatorname{det}\left(I-\lambda S_{\boldsymbol{u}}\right)-1\right| \leqslant\left(2^{m-1}-1\right) \frac{|\lambda|}{\tau(\partial \Omega)} .
\end{gathered}
$$

Proof. These follow immediately by writing $\operatorname{det}\left(I-\lambda S_{\boldsymbol{u}}\right)$ as the product of $1-\lambda \kappa_{j}$, where $\kappa_{j}$ are the principal curvatures (in particular, $\left|\kappa_{j}\right| \leqslant 1 / \tau(\partial \Omega)$ so $\left.\left|\lambda \kappa_{j}\right| \leqslant 1\right)$.

One use of these bounds is the following lemma, which allows Taylor's theorem in the direction normal to $\partial \Omega$ to be written with straightforward error terms, rather than using an awkward bound like

$$
\int_{\partial \Omega} \sup _{\lambda \in(-t, t)}|\nabla a(\boldsymbol{u}+\lambda \boldsymbol{n}(\boldsymbol{u}))| \mathrm{d} \boldsymbol{u} .
$$


Lemma 4.11. Let $t \leqslant \tau(\partial \Omega) / 2$, and let $g$ be a function on $\operatorname{tub}(\partial \Omega, t)$. For each $\boldsymbol{z} \in \operatorname{tub}(\partial \Omega, t)$ set $\boldsymbol{u}:=\boldsymbol{z}-\delta_{\Omega}(\boldsymbol{z}) \boldsymbol{n}(\boldsymbol{z}) \in \partial \Omega$. Set

$$
T:=\int_{\operatorname{tub}(\partial \Omega, t)}|a(z)-a(\boldsymbol{u})||g(z)| \mathrm{d} z .
$$

Then we have

$$
\begin{aligned}
& T \leqslant 3^{m-1}\left(\|\nabla a\|_{L^{1}(\partial \Omega)}+\|\nabla(\nabla a)\|_{L^{1}\left(\mathbb{R}^{m}\right)}\right) \sup _{\boldsymbol{u} \in \partial \Omega} \int_{-t}^{t}|\lambda g(\boldsymbol{u}+\lambda \boldsymbol{n}(\boldsymbol{u}))| \mathrm{d} \lambda, \\
& T \leqslant 3^{m-1}\|\nabla a\|_{L^{1}\left(\mathbb{R}^{m}\right)} \sup _{\boldsymbol{u} \in \partial \Omega} \int_{-t}^{t}|g(\boldsymbol{u}+\lambda \boldsymbol{n}(\boldsymbol{u}))| \mathrm{d} \lambda .
\end{aligned}
$$

Proof. By Lemma 4.8 and Lemma 4.10 we have

$$
T \leqslant\left(\frac{3}{2}\right)^{m-1} \int_{\partial \Omega} \int_{-t}^{t}|a(\boldsymbol{u}+\lambda \boldsymbol{n}(\boldsymbol{u}))-a(\boldsymbol{u})||g(\boldsymbol{u}+\lambda \boldsymbol{n}(\boldsymbol{u}))| \mathrm{d} \lambda \mu_{m-1}(\mathrm{~d} \boldsymbol{u}) .
$$

Applying Taylor's theorem to $a$ in the normal direction, we find

$a(\boldsymbol{u}+\lambda \boldsymbol{n}(\boldsymbol{u}))-a(\boldsymbol{u})=\lambda \boldsymbol{n}(\boldsymbol{u}) \cdot \nabla a(\boldsymbol{u})+\int_{0}^{1}(1-s) \lambda^{2}(\boldsymbol{n}(\boldsymbol{u}) \cdot \nabla)^{2} a(\boldsymbol{u}+s \lambda \boldsymbol{n}(\boldsymbol{u})) \mathrm{d} s$.

But changing variables $s^{\prime}=\lambda s$ for $|\lambda|<t$ we have

$\left|\int_{0}^{1}(1-s) \lambda^{2}(\boldsymbol{n}(\boldsymbol{u}) \cdot \nabla)^{2} a(\boldsymbol{u}+s \lambda \boldsymbol{n}(\boldsymbol{u})) \mathrm{d} s\right| \leqslant \int_{-t}^{t}\left|\lambda(\boldsymbol{n}(\boldsymbol{u}) \cdot \nabla)^{2} a\left(\boldsymbol{u}+s^{\prime} \boldsymbol{n}(\boldsymbol{u})\right)\right| \mathrm{d} s^{\prime}$,

so using Lemma 4.8 and Lemma 4.10 again (this time on $\mu_{m-1}(\mathrm{~d} \boldsymbol{u}) \mathrm{d} s^{\prime}$ rather than $\left.\mu_{m-1}(\mathrm{~d} \boldsymbol{u}) \mathrm{d} \lambda\right)$ gives the first inequality.

The second inequality follows in exactly the same way, except using one less term of the Taylor expansion.

The following two results are used in the composition step (\$3.2).

Lemma 4.12. For all $z \in \operatorname{tub}(\partial \Omega, \tau(\partial \Omega) / 2)$ we have the operator norm bound

$$
|\nabla \boldsymbol{n}(z)| \leqslant \frac{2}{\tau(\partial \Omega)} .
$$

Proof. This follows from Lemma 4.9 using that each $\left|\kappa_{j}\right| \leqslant 1 / \tau(\partial \Omega)$.

Lemma 4.13. Let $z \in \mathbb{R}^{m}, W \in \mathcal{S}\left(\mathbb{R}^{m}\right)$. Let $\Omega \subseteq \mathbb{R}^{m}$ have boundary satisfying $\tau(\partial \Omega) \geqslant 1$. Then

$$
\int_{\partial \Omega}|U(z-\boldsymbol{u})| \mu_{m-1}(\mathrm{~d} \boldsymbol{u}) \leqslant C_{d, U},
$$

where $C_{d, U}$ is a finite constant depending only on $d$ and $U$ (not on $z$ or $\Omega$ ). 
Proof. Set $\tilde{U}(\boldsymbol{u}):=\sup _{\boldsymbol{x} \in B(\boldsymbol{u}, 1 / 2)}|U(\boldsymbol{x})|$. Then

$$
\begin{aligned}
& \int_{\partial \Omega}|U(z-\boldsymbol{u})| \mu_{m-1}(\mathrm{~d} \boldsymbol{u}) \\
& \quad \leqslant 2^{m-1} \int_{-1 / 2}^{1 / 2} \int_{\partial \Omega}|U(z-\boldsymbol{u})| \operatorname{det}\left(I-\lambda S_{\boldsymbol{u}}\right) \mu_{m-1}(\mathrm{~d} \boldsymbol{u}) \mathrm{d} \lambda \\
& \quad \leqslant 2^{m-1} \int_{\operatorname{tub}(\partial \Omega, 1 / 2)} \tilde{U}\left(z-z^{\prime}\right) \mathrm{d} z^{\prime} \\
& \quad \leqslant 2^{m-1} \int_{\mathbb{R}^{m}} \tilde{U}\left(z^{\prime}\right) \mathrm{d} z^{\prime} .
\end{aligned}
$$

The following lemma is used in the trace asymptotics $(\$ 3.3)$ to show that certain integrands are concentrated close to the boundary of $\Omega$.

Lemma 4.14. Let $V \in L^{1}\left(\mathbb{R}^{m}\right)$, let $k \in \mathbb{N}_{0}$, and let $t<\tau(\partial \Omega)$. Then for all $\boldsymbol{u} \in \partial \Omega$ we have

$$
\begin{gathered}
\int_{-t}^{t}\left|\lambda^{k} V * \chi_{\Omega}(\boldsymbol{u}+\lambda \boldsymbol{n}(\boldsymbol{u})) V * \chi_{\Omega^{\mathrm{c}}}(\boldsymbol{u}+\lambda \boldsymbol{n}(\boldsymbol{u}))\right| \mathrm{d} \lambda \\
\quad \leqslant \frac{2}{k+1} \int_{\mathbb{R}^{m}}\left|V\left(\boldsymbol{z}^{\prime}\right)\right| \mathrm{d} \boldsymbol{z}^{\prime} \int_{\mathbb{R}^{m}}\left|\boldsymbol{z}^{\prime}\right|^{k+1}\left|V\left(\boldsymbol{z}^{\prime}\right)\right| \mathrm{d} \boldsymbol{z}^{\prime} .
\end{gathered}
$$

Proof. For $z \in \Omega$ we have $\Omega^{\mathrm{c}} \subseteq B(z, \operatorname{dist}(z, \partial \Omega))^{\mathrm{c}}$, so

$$
\left|V * \chi_{\Omega}(z)\right| \leqslant V_{\mathrm{rad}}(0), \quad\left|V * \chi_{\Omega^{\mathrm{c}}}(z)\right| \leqslant V_{\mathrm{rad}}(\operatorname{dist}(z, \partial \Omega)),
$$

where

$$
V_{\mathrm{rad}}(\lambda):=\int_{\left|z^{\prime}\right| \geqslant \lambda}\left|V\left(z^{\prime}\right)\right| \mathrm{d} z^{\prime} .
$$

Similar relationships hold for $z \in \Omega^{\mathrm{c}}$. But for $|\lambda|<\tau(\partial \Omega)$ we have

$$
\operatorname{dist}(\boldsymbol{u}+\lambda \boldsymbol{n}(\boldsymbol{u}), \partial \Omega)=|\lambda|,
$$

so the integral in the lemma statement is bounded by

$$
2 V_{\operatorname{rad}}(0) \int_{0}^{t}|\lambda|^{k} V_{\operatorname{rad}}(\lambda) \mathrm{d} \lambda .
$$

Interchanging the order of integration (between $\mathrm{d} \lambda$ and $\mathrm{d} z^{\prime}$ ) gives the result. 


\section{References}

[1] F. A. Berezin, Wick and anti-Wick operator symbols. Mat. Sb. (N.S.) 86(128) (1971), 578-610. In Russian. English transl., Math. USSR. Sb. 15 (1971) 577-606. MR 0291839 Zbl 0247.47018

[2] P. Boggiatto, E. Cordero, and K. Gröchenig, Generalized anti-Wick operators with symbols in distributional Sobolev spaces. Integral Equations Operator Theory $\mathbf{4 8}$ (2004), no. 4, 427-442. MR 2047590 Zbl 1072.47045

[3] A. Böttcher and B. Silbermann, Analysis of Toeplitz operators. Springer, Berlin, second edition, 2006. MR 1071374 Zbl 0732.47029

[4] A. Boulkhemair, $L^{2}$ estimates for Weyl quantization. J. Funct. Anal. 165 (1999), no. 1, 173-204. MR 1696697 Zbl 0934.35217

[5] I. Daubechies, Time-frequency localization operators: a geometric phase space approach. IEEE Trans. Inform. Theory 34 (1988), no. 4, 605-612. MR 0966733 Zbl 0672.42007

[6] F. De Mari, H. G. Feichtinger, and K. Nowak, Uniform eigenvalue estimates for timefrequency localization operators. J. London Math. Soc. (2) 65 (2002), no. 3, 720-732. MR 1895743 Zbl 1033.47021

[7] M. Dimassi and J. Sjöstrand, Spectral asymptotics in the semi-classical limit. London Mathematical Society Lecture Note Series, 268. Cambridge University Press, Cambridge, 1999. MR 1735654 Zbl 0926.35002

[8] H. G. Feichtinger and K. Nowak, A Szegö-type theorem for Gabor-Toeplitz localization operators. Michigan Math. J. 49 (2001), no. 1, 13-21. MR 1827072 Zbl 1010.47021

[9] G. B. Folland, Harmonic analysis in phase space. Annals of Mathematics Studies, 122. Princeton University Press, Princeton, N.J., 1989. MR 0983366 Zbl 0682.43001

[10] D. Gilbarg and N. S. Trudinger, Elliptic partial differential equations of second order. Grundlehren der Mathematischen Wissenschaften, 224. Springer, Berlin etc., 1977. MR 0473443 Zbl 0361.35003

[11] A. Gray, Tubes. $2^{\text {nd }}$ ed. With a preface by V. Miquel. Progress in Mathematics, 221. Birkhäuser, Basel, 2004. MR 2024928 Zbl 1048.53040

[12] U. Grenander and G. Szegó, Toeplitz forms and their applications. California Monographs in Mathematical Sciences. University of California Press, Berkeley and Los Angeles, CA, 1958. MR 0094840 Zbl 0080.09501

[13] K. Gröchenig, Foundations of time-frequency analysis. Applied and Numerical Harmonic Analysis. Birkhäuser Boston, Inc., Boston, MA, 2001. MR 1843717 Zbl 0966.42020

[14] A. Martinez, An introduction to semiclassical and microlocal analysis. Universitext. Springer, New York, 2002. MR 1872698 Zbl 0994.35003

[15] H. M. Ozaktas, Z. Zalevsky, and M. A. Kutay, The fractional Fourier transform with applications in optics and signal processing. Series in Pure and Applied Optics. John Wiley \& Sons, Chichester, 2001. 
[16] J. Ramanathan and P. Topiwala, Time-frequency localization and the spectrogram. Appl. Comput. Harmon. Anal. 1 (1994), no. 2, 209-215. MR 1310645 Zbl 0802.42030

[17] D. Robert, Autour de l'approximation semi-classique. Progress in Mathematics, 68. Birkhäuser Boston, Inc., Boston, MA, 1987. MR 0897108 Zbl 0621.35001

[18] R. Roccaforte, Asymptotic expansions of traces for certain convolution operators. Trans. Amer. Math. Soc. 285 (1984), no. 2, 581-602. MR 0752492 Zbl 0596.47018

[19] R. Roccaforte, Volume estimates and spectral asymptotics for a class of pseudodifferential operators. J. Pseudo-Differ. Oper. Appl. 4 (2013), no. 1, 25-43. MR 3022799 Zbl 1260.35265

[20] A. V. Sobolev, Wiener-Hopf operators in higher dimensions: the Widom conjecture for piece-wise smooth domains. Integral Equations Operator Theory 81 (2015), no. 3, 435-449. MR 3311886 Zbl 1307.47054

[21] A. V. Sobolev, Pseudo-differential operators with discontinuous symbols: Widom's conjecture. Mem. Amer. Math. Soc. 222 (2013), no. 1043. MR 3076415 Zbl 1297.47054

[22] G. Szegó, Ein Grenzwertsatz über die Toeplitzschen Determinanten einer reellen positiven Funktion. Math. Ann. 76 (1915), no. 4, 490-503. MR 1511838 JFM 45.0518.02

[23] G. Szegó, On certain Hermitian forms associated with the Fourier series of a positive function. Comm. Sém. Math. Univ. Lund [Medd. Lunds Univ. Mat. Sem.] (1952), Tome Supplémentaire, 228-238. MR 0051961 Zbl 0048.04203

[24] H. Widom, On a class of integral operators with discontinuous symbol. In I. Gohberg (ed.), Toeplitz centennial. Proceedings of the Toeplitz Memorial Conference on Operator Theory held in Tel Aviv, May 11-15, 1981. Dedicated to the 100th anniversary of the birth of Otto Toeplitz. Operator Theory: Advances and Applications, 4. Birkhäuser Verlag, Basel and Boston, MA, 1982, 477-500. MR 0669925 MR 0669898 (collection) Zbl 0483.47037 Zbl 0474.00006 (collection)

[25] H. Widom, Asymptotic expansions for pseudodifferential operators on bounded domains. Lecture Notes in Mathematics, 1152. Springer, Berlin, 1985. MR 0811855 Zbl 0582.35002

Received April 18, 2014

James P. Oldfield, Department of Mathematics, University College London, Gower Street, London WC1E 6BT, U.K.

e-mail: james.oldfield.09@ucl.ac.uk 